\title{
ON THE SOLVABILITY \\ OF A TWO-DIMENSIONAL WAVE-BODY INTERACTION PROBLEM
}

BY

\author{
G. A. ATHANASSOULIS AND C. G. POLITIS
}

National Technical University of Athens, Athens, Greece

\begin{abstract}
The two-dimensional, deep-water, wave-body interaction problem for a single-hulled body, floating on the free surface of an ideal liquid, is considered. The body boundary may be nonsmooth and may intersect the free surface at arbitrary angles. The existence of a unique solution representable by a multipole-series expansion is proved for all but a discrete set of oscillation frequencies. The proof is based on the property of the associated multipoles to be a basis of the space $L^{P}(-\pi, 0)$, $1<p \leq 2$. Strict estimates of the form $D_{n}=O\left(n^{-\alpha}\right)$ are also obtained for the coefficients of the multipole-series expansion for piecewise smooth $(0<\alpha<2)$ and smooth $(\alpha=2)$ body boundaries.
\end{abstract}

1. Introduction. Consider an infinitely long, horizontal cylinder floating on the free surface of an unbounded, infinitely deep, incompressible and inviscid liquid. In this paper we are concerned with the solvability (well-posedness) of the boundary-value problems arising when the floating body is forced, by an incident harmonic wave or by an external force, to perform time-harmonic oscillations of small amplitude about its position of stable hydrostatic equilibrium.

The uniqueness question for the wave-floating body interaction problem has been studied by means of two, essentially different, methodologies. The first one is of geometric character and appropriately uses Green's theorem to ensure that the only possible solution of the homogeneous problem is the trivial one. Such an approach was inaugurated by John [18] in 1950 (see also Lenoir [24], who reworked the twodimensional case, and Kleinman [21]). Recently, Simon and Ursell [33] essentially generalized John's uniqueness theorem in a manner permitting them to consider floating and/or submerged bodies of quite a general shape. In general, the uniqueness results obtained via this methodology are valid for all wavelengths but have suffered, up to now, from some geometrical restrictions concerning the shape of the floating body. Having established uniqueness, the well-posedness of the problem can be deduced by using various methods, such as the integral-equation formulation [18, $21,22]$ or the limiting absorption principle [11, 24, 25]. 
The second methodology for tackling the uniqueness (and, in fact, the wellposedness) question is of analytic character and proceeds as follows. The problem is extended to the complex frequency domain and it is properly reformulated as a Fredholm functional equation of the second kind of the form

$$
(B+T(\lambda)) u=v,
$$

where $B$ is a bounded operator and $T(\lambda)$ is a compact operator analytically dependent upon the complex wavenumber $\lambda$. Then, the Fredholm theory for analytic families of compact operators is invoked ensuring the unique solvability of Eq. (1.1) for all but a discrete set of values of $\lambda$. (Note, however, that the well-posedness remains valid at these exceptional frequencies too, provided that uniqueness can be established there by any manner; see Remark 4.1.) The first author who used this method to deduce uniqueness of the wave-body interaction problem was apparently Beale $[9$, Theorem 5.4]. See also Vullierme-Ledard [43], who treated the infinite-depth case for either a floating or a submerged body. In these works the body boundary is assumed to be smooth. Recently, Athanassoulis [3] has used a multipole-series expansion [2] to obtain a functional-equation of the form (1.1) for the two-dimensional problem. The geometric assumptions used in the latter work were that the wetted surface of the floating body is smooth and it intersects the free surface perpendicularly. These unnecessarily restrictive assumptions will be removed in the present paper.

In the present paper the wetted surface of the floating body may be nonsmooth and may intersect the free surface at arbitrary angles (different from 0 and $\pi$ ). More precisely, it can belong to the class $W^{1, p}, p>1$, (see Def. 2.1), where the class index $p$ is related to the maximum slope-incremental discontinuity of the wetted-surface tangent. To obtain the well-posedness results, the liquid domain is conformally transformed onto the lower exterior semidisk $\{\operatorname{Im} \zeta \leq 0,|\zeta|>1\}$, and the problem is reformulated in the transformed plane. Then, taking into account the boundary behaviour of the conformal mapping function and its derivative (Theorem 2.1), and using the multipole-expansion representation of the solution (Theorem 3.1), we prove that the traces of the multipoles onto the lower semicircle form a basis in the space $L^{p^{*}}(-\pi, 0), p^{*}=\min (p, 2)$, (Theorem 4.3). This result is used to prove the existence of a unique wave potential $\mathbf{F}\left(\zeta ; k_{0}\right)$ representable by means of a multipole-series convergent up to and including the body boundary (Theorem 5.1). It is also proved that, in general, the coefficients $D_{m}$ of the multipole-series expansion of $d \mathbf{F}\left(\zeta ; k_{0}\right) / d \zeta$ satisfy the relation $\sum\left|D_{m}\right|^{q^{*}}<\infty, q^{*}=p^{*} /\left(p^{*}-1\right)$. For piecewise smooth boundaries the above estimate for $D_{m}$ is improved to $D_{m}=O\left(m^{-i}\right)$, where $\alpha \pi$ is the smaller exterior angle of the double-body boundary (Theorem 6.1). For smooth boundaries we have $D_{m}=O\left(\mathrm{~m}^{-2}\right)$, which is inconsistent with Ursell's result for the floating semicircle [42]. The latter estimate cannot be further improved however smooth the boundary is (Theorem 6.2).

2. Notation, terminology and some preliminary results. A Cartesian coordinate system $O_{2} x_{3}$ is introduced with the $x_{2}$-axis lying on the mean free surface $\partial D_{F}$, the $x_{3}$-axis directed vertically upwards and the origin $O$ fixed inside the floating body. A point in the plane $O x_{2} x_{3}$ (the physical plane) is denoted by $x=\left(x_{2}, x_{3}\right)$ 
or $w=x_{2}+i x_{3}$, in the complex notation. The mean liquid domain, the mean position of the immersed part of the floating body and the mean position of the wetted surface of the body are denoted by $D^{-}, D_{B}^{-}$, and $\partial D_{B}^{-}$, while their mirror images with respect to the $x_{2}$-axis are denoted by $D^{+}, D_{B}^{+}$, and $\partial D_{B}^{+}$, respectively. The pointsets $D_{B}=D_{B}^{-} \cup D_{B}^{+}, \partial D_{B}=\partial D_{B}^{-} \cup \partial D_{B}^{+}$, and $D=D^{-} \cup D^{+} \cup \partial D_{F}$ will be referred to as the double body, the double-body boundary, and the exterior domain, correspondingly. The unit normal on $\partial D_{B}^{-}$, directed inwards with respect to the liquid, is denoted by $n=\left(n_{2}, n_{3}\right)$; see Fig. 1 .

The pointsets $D^{-}, D^{+}$, and $D$ are considered topologically open, whilst $D_{B}^{-}, D_{B}^{+}$, and $D_{B}$ are considered topologically closed. The points of intersection of the body boundary and the free surface $B_{\ell}, B_{r}$ are considered to belong to $\partial D_{B}^{-}$and $\partial D_{B}^{+}$, but not to $\partial D_{F}$.

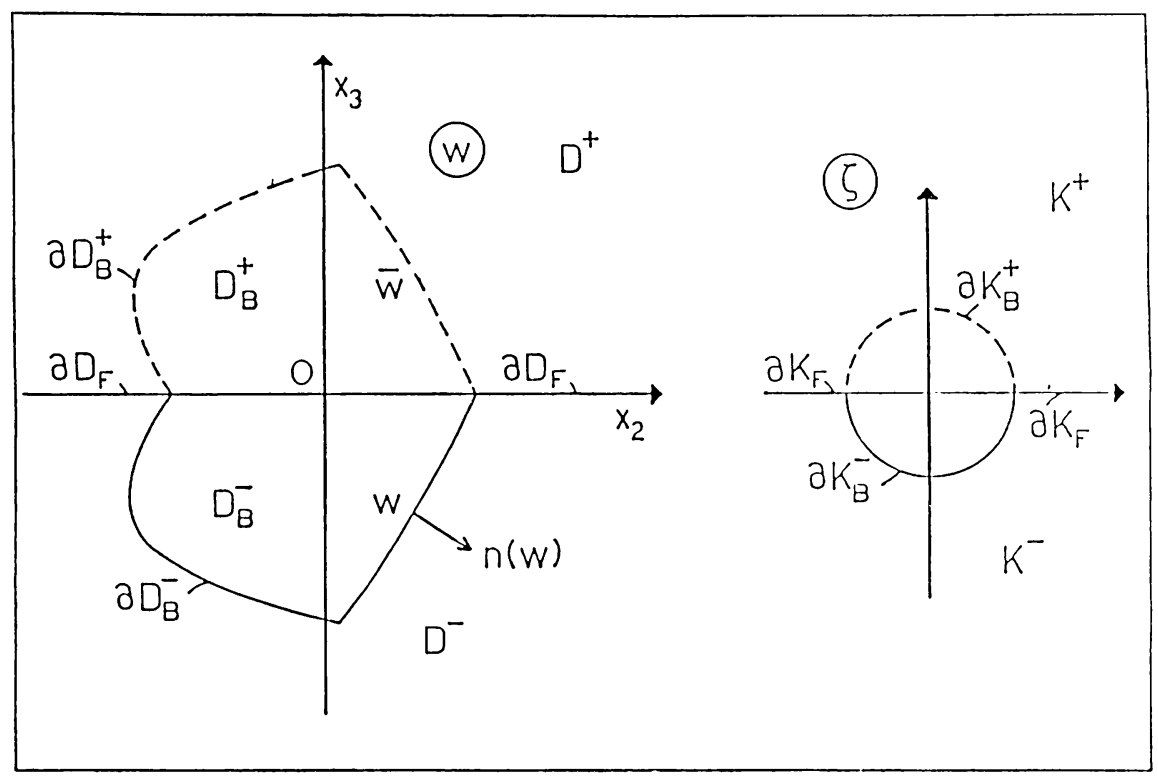

FIG. 1

For the exact mathematical formulation of the boundary-value problem which we intend to study, we need to characterize exactly the double-body boundary $\partial D_{B}$. We recall that a closed rectifiable Jordan curve $\partial D_{B}$ is said to be quasi-smooth [29], or of bounded arc-length-chord-length ratio [45], if

$$
\ell\left(w_{1}, w_{2}\right) /\left|w_{1}-w_{2}\right| \leq b, \quad 1<b<\infty, w_{1}, w_{2} \in \partial D_{B},
$$

where $\ell\left(w_{1}, w_{2}\right)$ is the length of the shorter arc of $\partial D_{B}$ between $w_{1}$ and $w_{2}$. Also, a closed rectifiable Jordan curve $\partial D_{B}$ is called of bounded rotation [31, p. 225], if the forward half-tangent exists at every point of $\partial D_{B}$, and the tangent angle $\tau(s)$ which it makes with a fixed direction (the slope) may be defined as a function of bounded variation of the arc length $s, 0 \leq s \leq L$. Let $v_{+}(s)$ be the positive variation function 
of $\tau(s)$, and define the (positive) jump index $\alpha$ of $\partial D_{B}$ by

$$
\alpha=\sup _{0 \leq s \leq L}\left[v_{+}(s+0)-v_{+}(s-0)\right] / \pi .
$$

Geometrically, the jump index is related to the maximum slope-incremental discontinuity. See below, Eq. (2.3).

Definition 2.1. (Cf. [45, p. 203].) We shall say that the boundary $\partial D_{B}$ belongs to the class $W^{1, p}$ if: (i) it is quasi-smooth, and (ii) it is of bounded rotation, with jump index $\alpha<1 / p$. The positive constant $p$ will be referred to as the class index.

An interesting special case of boundaries belonging to the class $W^{1, p}$ are those which are sectionally smooth and satisfy condition (2.1). In this case the tangent angle $\tau(s)$ is continuous except for a finite number of points, at which $\partial D_{B}$ has vertices with exterior angles $a_{\sigma} \pi, 0<a_{\sigma}<2, \sigma=1,2, \ldots, \Sigma$. The jump index $\alpha$ is now given by

$$
\alpha=\max _{1 \leq \sigma \leq \Sigma}\left[1-a_{\sigma}, 0\right]=\max \left[1-a_{*}, 0\right]<1,
$$

where $a_{*}=\min \left[a_{1}, a_{2}, \ldots, a_{\Sigma}\right]$. In this case the class index $p$ is related to the smaller exterior angle $a_{*} \pi$ as follows

$$
a_{*} \pi>(p-1) \pi / p
$$

In treating the wave-body interaction problem by means of the multipole-expansion method use is made of the conformal mapping function (cmf) $f(\zeta)$, which transforms the exterior unit disk $K$ onto the exterior of the double-body boundary $D$. Such a function may be chosen so that the lower exterior unit semi-disk $K^{-}$, the lower unit semi-circle $\partial K_{B}^{-}$, and the part $\partial K_{F}$ of the real axis outside the unit circle of the $\zeta$-plane correspond to the pointsets $D^{-}, \partial D_{B}^{-}$, and $\partial D_{F}$ of the $w$-plane, respectively; see Fig. 1.

Assuming that $\partial D_{B}$ is a closed, rectifiable Jordan curve, the $\mathrm{cmf} f(\zeta)$ admits of a Laurent expansion of the form

$$
f(\zeta)=\sum_{n=1}^{\infty} c_{n} \zeta^{2-n}, \quad c_{n} \in \mathbb{R}, \quad c_{1}>0
$$

which converges absolutely and uniformly up to and including the unit circle $\partial K_{B}$. The function $w(\theta)=f\left(e^{i \theta}\right),-\pi \leq \theta \leq \pi$, is absolutely continuous on $\partial K_{B}$ and it defines a parametric representation of $\partial D_{B}$. The derivatives $f^{\prime}\left(e^{i \theta}\right)$ and $w^{\prime}(\theta)=$ $d f\left(e^{i \theta}\right) / d \theta$ exist almost everywhere (a.e.) on $\partial K_{B}$, belong to $L^{1}\left(\partial K_{B}\right)$, and satisfy the relation

$$
w^{\prime}(\theta)=i e^{i \theta} d f\left(e^{i \theta}\right) / d \zeta \neq 0, \infty, \quad \text { a.e. on } \partial K_{B} .
$$

Moreover, the unit normal $n(w)=n_{2}+i n_{3}$ is defined a.e. on $\partial D_{B}$ and is expressed in terms of $f(\zeta)$ by means of the equation

$$
n(w)=\zeta f^{\prime}(\zeta) /\left|f^{\prime}(\zeta)\right|, \quad w=f(\zeta), \zeta=e^{i \theta} .
$$

The proofs of the above propositions may be found in $[14,28,37]$. 
The boundary behaviour of $f^{\prime}(\zeta)$ and $w^{\prime}(\theta)$ for domains whose boundaries belong to the class $W^{1, p}$ is characterized by means of

Theorem 2.1. Let $\partial D_{B} \in W^{1, p}$. Then $f^{\prime}(\zeta)$ belongs to the Hardy space $H^{p}(K)$ and the functions $f^{\prime}\left(e^{i \theta}\right)$ and $w^{\prime}(\theta)$ belong to $L^{p}(-\pi, \pi)$.

This theorem is sharp in the sense that the space $H^{p}(K)$ is the best possible for the class $W^{1, p}$. Indeed, there exist curves (e.g., polygons) with jump index $\alpha=1 / p$, but for which $f^{\prime}(\zeta) \notin H^{p}(K)$. Theorem 2.1 has been proved by Warschawski and Schober [45] for bounded simply-connected domains, but it may be easily extended to exterior domains [26, 30,32]. It should be emphasized that the above seemingly simple theorem is a deep and difficult to obtain result, and constitutes the key tool for treating the wave-body interaction problem when the body boundaries are nonsmooth. It permits us to obtain all the required analytic properties of the derivative of the conformal mapping function $f^{\prime}\left(e^{i \theta}\right), \theta \in[-\pi, \pi]$, without resorting to its series expansion, which, in this case, may converge slowly or even diverge at some corner points.

Finally, we note that in the present work two noninteracting, functionally different, imaginary units $i$ and $j$ are used, generating the sets of $i$-complex and $j$-complex numbers, denoted by $\mathbb{C}_{i}$ and $\mathbb{C}_{j}$, respectively. The unit $i$ is used for the complexification of the physical plane, while the unit $j$ is used for factoring out the time-harmonic dependence. Formal products of $i$ - and $j$-complex numbers also occur, generating the set of $i j$-complex numbers, denoted by $\mathbb{C}_{i j}$. For a complete study of the algebraic and topological structure of $\mathbb{C}_{i j}$ see [2, Appendix I].

3. The multipole expansion method. Under the assumptions stated in Sec. 1, the liquid motion may be described by a velocity potential $\Phi_{R}(x ; t)=\operatorname{Re}_{j}\left\{j \omega \Phi(x ; \omega) e^{j \omega t}\right\}$, where $\omega$ is the frequency of oscillation. In treating the wave-body interaction problem, two different physical problems are to be considered, namely, the radiation problem and the diffraction problem [46, Sec. 19]. In either case the potential $\Phi=\Phi(x ; \omega)$ has to satisfy the Laplace equation, the boundary conditions

$$
\begin{gathered}
k_{0} \Phi-\Phi_{, 3}=0, \quad \text { on } \partial D_{F}, \quad \partial \Phi / \partial n=V\left(x ; k_{0}\right), \quad \text { on } \partial D_{B}^{-}, \\
\left|\Phi_{, 2}\right|,\left|\Phi_{, 3}\right| \rightarrow 0, \quad \text { as } x_{3} \rightarrow-\infty,
\end{gathered}
$$

and the radiation condition

$$
\Phi_{, 2} \mp j k_{0} \Phi \rightarrow 0, \quad \text { as } x_{2} \rightarrow \pm \infty,
$$

where $k_{0}=\omega^{2} / g$ is the wavenumber, $g$ is the acceleration of the gravity, $\partial / \partial n$ denotes differentiation along the normal $n=\left(n_{2}, n_{3}\right)$ of $\partial D_{B}^{-}$, and $j \omega V\left(x ; k_{0}\right)$ is the normal boundary velocity on $\partial D_{B}^{-}$, depending on the physical problem considered. Since, in general, we assume that $\partial D_{B} \in W^{1, p}$ for some $p>1$, the unit normal $n$ is defined a.e. on $\partial D_{B}^{-}$.

Using the $i j$-complex wave potential $F\left(w ; k_{0}\right)=\Phi\left(x ; k_{0}\right)+i \Psi\left(x ; k_{0}\right)$, where $\Psi\left(x ; k_{0}\right)$ is the $j$-complex amplitude of the stream function, the body-boundary condition (3.1b) takes the form

$$
\operatorname{Re}_{i}\left\{n(w) F^{\prime}\left(w ; k_{0}\right)\right\}=\operatorname{Re}_{i}\left\{\bar{v}\left(w ; k_{0}\right) n(w)\right\}, \quad \text { for a.a. } w \in \partial D_{B}^{-},
$$


where "a.a." stands for "almost all," the prime denotes differentiation with respect to the space variable, the overbar denotes $i$-complex conjugation, and $v\left(w ; k_{0}\right)$ is the $i j$-complex body-velocity field. In the case of rigid-mode oscillations of the floating body with amplitudes $\xi_{2}$ (sway), $\xi_{3}$ (heave), and $\xi_{4}$ (roll), we have

$$
v\left(w ; k_{0}\right)=\left[\xi_{2}+i \xi_{3}+i \xi_{4} w\right]
$$

while in the case of the diffraction problem we have

$$
v\left(w ; k_{0}\right)=A(j-i) \exp \left(i k_{0} \bar{w}\right)
$$

where $A$ is the amplitude of the incident wave.

Consider now the conformal mapping function $f(\zeta)$ introduced in Sec. 2. Applying the change of variable $w=f(\zeta)$ we transform $F\left(w ; k_{0}\right)$ into the potential $\mathbf{F}\left(\zeta ; k_{0}\right)=F\left(f(\zeta) ; k_{0}\right)$, defined on the lower exterior semidisk $K^{-}$. Using $\mathbf{F}\left(\zeta ; k_{0}\right)$ and the geometric relations (2.6) and (2.7) we reformulate the boundary-value problem (3.1) in the following form:

Problem $\mathbf{P}\left(\zeta ; k_{0}\right)$. Let $\partial D_{B} \in W^{1, p}, p>1$ and $k_{0} \in \mathbb{C}_{j}-\{0\}$. Find an $i j$-complex function $\mathbf{F}\left(\zeta ; k_{0}\right)$, analytic with respect to $\zeta \in K^{-}(\zeta$-analytic $)$ and satisfying the conditions

$$
\begin{gathered}
\operatorname{Im}_{i}\left\{\mathbf{F}^{\prime}\left(\zeta ; k_{0}\right)+i k_{0} f^{\prime}(\zeta) \mathbf{F}\left(\zeta ; k_{0}\right)\right\}=0, \quad \zeta \in \partial K_{F}, \\
\operatorname{Re}_{i}\left\{\zeta \mathbf{F}^{\prime}\left(\zeta ; k_{0}\right)\right\}=\operatorname{Re}_{i}\left\{\overline{\boldsymbol{v}}\left(\zeta ; k_{0}\right) \zeta f^{\prime}(\zeta)\right\}, \quad \text { for a.a. } \zeta \in \partial K_{B}^{-}, \\
\left|\mathbf{F}^{\prime}\left(\zeta ; k_{0}\right)\right|_{c_{i j} \rightarrow 0,} \text { as } \operatorname{Im}_{i}\{\zeta\} \rightarrow-\infty, \\
\mathbf{F}\left(\zeta ; k_{0}\right) \rightarrow \Lambda^{ \pm}\left(k_{0}\right)(1 \mp i j) k_{0} \exp \left(-i k_{0} f^{\prime}(\infty) \zeta\right), \quad \Lambda^{ \pm}\left(k_{0}\right) \in \mathbb{C}_{j}, \quad \operatorname{Re}_{i}\{\zeta\} \rightarrow \pm \infty,
\end{gathered}
$$

where $v\left(\zeta ; k_{0}\right)=v\left(f(\zeta) ; k_{0}\right)$.

Since the right-hand side of $(3.5 \mathrm{~b})$ belongs to $L^{p}\left(\partial K_{B}^{-}\right), p>1$, the equality should be understood in the $L^{p}$-sense. Condition $(3.5 \mathrm{~d})$ is an alternative form of the radiation condition (3.1d); see [2].

In the present work, the problem $\mathbf{P}\left(\zeta ; k_{0}\right)$ will be treated by means of the multipoleexpansion method. This method has been first introduced by Ursell in 1949 [38, 39] (see also [40, 42]) for smooth floating bodies with a vertical axis of symmetry, and it was extended to smooth nonsymmetric bodies by Wehausen [47], Count [10], and Athanassoulis [2, 3]. Here, the applicability of the multipole-expansion method is confirmed for arbitrary (nonsmooth, nonsymmetric) floating bodies. All technical features of the multipole-series expansion used in this work are presented in

TheOREM 3.1. (The expansion Theorem [2].) Let $\partial D_{B}=\partial D_{B}^{-} \cup \partial D_{B}^{+}$be a closed Jordan curve. A function $\mathbf{F}\left(\zeta ; k_{0}\right)$ is $\zeta$-analytic, $\zeta \in K^{-}$, and satisfies $(3.5 \mathrm{a}, \mathrm{c}$, d) iff it may be represented in the form

$$
\mathbf{F}\left(\zeta ; k_{0}\right)=d_{0} \mathbf{G}_{0}\left(\zeta ; k_{0}\right)+d_{1} \mathbf{G}_{1}\left(\zeta ; k_{0}\right)+\sum_{m=2}^{\infty} d_{m} \mathbf{M}_{m}\left(\zeta ; k_{0}\right)
$$


where $d_{m} \in \mathbb{C}_{j}, m=0,1,2, \ldots$,

$$
\begin{gathered}
\mathbf{M}_{m}\left(\zeta ; k_{0}\right)=\zeta^{-m}-i k_{0} \int_{\infty+i 0}^{\zeta} u^{-m} f^{\prime}(u) d u=\zeta^{-m}-i k_{0} \sum_{\ell=1}^{\infty} \frac{(2-\ell) c_{\ell} \zeta^{2-\ell-m}}{2-\ell-m} \\
\mathbf{G}_{m}\left(\zeta ; k_{0}\right)=G_{m}\left(f(\zeta) ; k_{0}\right), \quad m=0,1,3, \ldots \\
G_{0}\left(w ; k_{0}\right)=(1-i j) \exp \left(-i k_{0} w\right)-j F_{0}\left(w ; k_{0}\right) / \pi \\
G_{1}\left(w ; k_{0}\right)=(1-i j) k_{0} \exp \left(-i k_{0} w\right)-F_{1}\left(w ; k_{0}\right) / \pi
\end{gathered}
$$

and

$$
F_{m}\left(w ; k_{0}\right)=\exp \left(-i k_{0} w\right) \int_{\infty+i 0}^{w} u^{-(m+1)} \exp \left(i k_{0} u\right) d u, \quad m=0,1 .
$$

The path of integration in Eqs. (3.7) and (3.10) is taken in the lower half-plane.

The function $F_{0}\left(w ; k_{0}\right)$ admits of the series representation, [15]

$$
F_{0}\left(w ; k_{0}\right)=\exp \left(-i k_{0} w\right)\left[\gamma+\ln \left(i k_{0} w\right)-i \pi+\sum_{n=1}^{\infty} \frac{\left(i k_{0} w\right)^{n}}{n \cdot n !}\right],
$$

where $\gamma$ is Euler's constant. This expression shows that $F_{0}\left(w ; k_{0}\right)$ is, for each $w \neq 0$, an analytic function of $k_{0} \in Q$, where $Q \subset \mathbb{C}_{j}-\{0\}$ is any open bounded simplyconnected region. Moreover, the function $k_{0} F_{0}\left(w ; k_{0}\right) \rightarrow 0$ as $k_{0} \rightarrow 0$, for each fixed $w \neq 0$. Since

$$
F_{1}\left(w ; k_{0}\right)=i k_{0} F_{0}\left(w ; k_{0}\right)-w^{-1}
$$

and

$$
d F_{m}\left(w ; k_{0}\right) / d w=w^{-(m+1)}-i k_{0} F_{m}\left(w ; k_{0}\right), \quad m=0,1,
$$

it follows that $F_{1}\left(w ; k_{0}\right)$ and $d F_{m}\left(w ; k_{0}\right) / d w$ are also analytic functions of $k_{0} \in Q$ and they remain bounded as $k_{0} \rightarrow 0$, for each $w \neq 0$.

Substituting the expansion (3.6) into the body-boundary condition (3.5b) and formally differentiating term-by-term we obtain

$$
\sum_{m=0}^{\infty} D_{m}\left(k_{0}\right) \phi_{m}\left(\theta ; k_{0}\right)=\mathbf{V}\left(\theta ; k_{0}\right), \quad \text { in } L^{p}(-\pi, 0),
$$

where

$$
\begin{gathered}
D_{m}=d_{m}, \quad m=0,1, \quad D_{m}=-m d_{m}, \quad m=2,3, \ldots, \\
\phi_{m}\left(\theta ; k_{0}\right)=\operatorname{Re}_{i}\left\{e^{i \theta} \mathbf{G}_{m}^{\prime}\left(e^{i \theta} ; k_{0}\right)\right\}=\operatorname{Re}_{i}\left\{H_{m}\left(e^{i \theta} ; k_{0}\right)\right\}, \quad m=0,1,
\end{gathered}
$$

with

$$
\begin{aligned}
H_{0}\left(e^{i \theta} ; k_{0}\right)= & (-1+i j) k_{0} w^{\prime}(\theta) \exp \left(-i k_{0} w(\theta)\right)+j k_{0} w^{\prime}(\theta) F_{0}\left(w(\theta) ; k_{0}\right) / \pi \\
& +i j w^{\prime}(\theta) /(\pi w(\theta)), \\
H_{1}\left(e^{i \theta} ; k_{0}\right)= & (-1+i j) k_{0}^{2} w^{\prime}(\theta) \exp \left(-i k_{0} w(\theta)\right)+i k_{0}^{2} w^{\prime}(\theta) F_{0}\left(w(\theta) ; k_{0}\right) / \pi \\
& -k_{0} w^{\prime}(\theta) /(\pi w(\theta))+i w^{\prime}(\theta) /\left(\pi w^{2}(\theta)\right),
\end{aligned}
$$




$$
\begin{aligned}
\phi_{m}\left(\theta ; k_{0}\right) & =\operatorname{Re}_{i}\left\{e^{i \theta} \mathbf{M}_{m}^{\prime}\left(e^{i \theta} ; k_{0}\right) / m\right\} \\
& =\operatorname{Re}_{i}\left\{e^{-i m \theta}+i k_{0} e^{-i m \theta} e^{i \theta} f^{\prime}\left(e^{i \theta}\right) / m\right\} \\
& =\cos (m \theta)+k_{0} g_{m}(\theta) / m, \quad m=2,3, \ldots,
\end{aligned}
$$

with

$$
g_{m}(\theta)=\operatorname{Re}_{i}\left\{e^{-i m \theta} w^{\prime}(\theta)\right\}=x_{2}^{\prime}(\theta) \cos (m \theta)+x_{3}^{\prime}(\theta) \sin (m \theta),
$$

and

$$
\mathbf{V}\left(\theta ; k_{0}\right)=\operatorname{Re}_{i}\left\{\overline{\boldsymbol{v}}\left(e^{i \theta} ; k_{0}\right) e^{i \theta} f^{\prime}\left(e^{i \theta}\right)\right\}=\operatorname{Re}_{i}\left\{-i \overline{\boldsymbol{v}}\left(e^{i \theta} ; k_{0}\right) w^{\prime}(\theta)\right\}
$$

In this way the question of the solvability of the problem $\mathbf{P}\left(\zeta ; k_{0}\right)$ has been reduced to the question of the expansibility of the forcing function $\mathbf{V}\left(\theta ; k_{0}\right)$ in terms of the functions $\phi_{m}\left(\theta ; k_{0}\right), m=0,1, \ldots$ In this connection, before proceeding any further, we should characterize these functions.

LeMmA 3.1. Let $\partial D_{B} \in W^{1, p}, 1<p \leq \infty$. Then,

(i) For any given $k_{0} \in \mathbb{C}_{j}$, the functions $\mathbf{V}\left(\theta ; k_{0}\right), g_{m}\left(\theta ; k_{0}\right), m \geq 2$, and $\phi_{m}\left(\theta ; k_{0}\right)$, $m \geq 0$, belong to the space $L^{p}(-\pi, 0)$;

(ii) For almost all $\theta \in[-\pi, 0]$, the above functions are analytic functions of $k_{0} \in \mathbb{C}_{j}-\{0\}$

(iii) The following inequalities hold true:

$$
\sum_{m=2}^{\infty}\left\|\phi_{m}\left(\theta ; k_{0}\right)-\cos (m \theta)\right\|_{L^{p}}^{p} \equiv\left|k_{0}\right| \sum_{m=2}^{\infty}\left\|\frac{g_{m}(\theta)}{m}\right\|_{L^{p}}^{p} \leq\left|k_{0}\right|\left\|w^{\prime}(\theta)\right\|_{L^{p}}^{p}\left(\sum_{m=2}^{\infty} m^{-p}\right) \text {. }
$$

Proof. (i) It is a straightforward consequence of Theorem 2.1 .

(ii) It is an obvious consequence of Eqs. (3.11)-(3.13) and (3.16)-(3.17).

(iii) This is a direct consequence of the fact that

$$
\left\|x_{2}^{\prime}(\theta) \cos (m \theta)+x_{3}^{\prime}(\theta) \sin (m \theta)\right\|_{L^{p}} \leq\left\|w^{\prime}(\theta)\right\|_{L^{p}},
$$

and the Theorem 2.1.

REMARK 3.1. It should be emphasized that the characterization ("smoothness") of the functions $\mathbf{V}\left(\theta ; k_{0}\right), g_{m}\left(\theta ; k_{0}\right), m \geq 2$, and $\phi_{m}\left(\theta ; k_{0}\right), m \geq 0$, given in the above lemma, is the best possible, in the sense that, for a boundary $\partial D_{B}$ with a jump index $\alpha$, these functions belong to $L^{p}(-\pi, 0)$ for all $p<1 / \alpha$, but they do not belong to $L^{1 / \alpha}(-\pi, 0)$.

We are now in a position to state the following alternative formulation of the problem $\mathbf{P}\left(\zeta ; k_{0}\right)$.

Problem $\mathbf{P}\left(\mathbf{D} ; k_{0}\right)$. Let $\partial D_{B}, k_{0}, \boldsymbol{v}\left(\zeta ; k_{0}\right)$ be as in Problem $\mathbf{P}\left(\zeta ; k_{0}\right)$. Find a $j$ complex sequence $\mathbf{D}\left(k_{0}\right)=\left\{D_{m}\left(k_{0}\right)\right\}_{0}^{\infty}$ such that Eq. (3.14) is satisfied in $L^{p}(-\pi, 0)$.

To clarify the method that will be used in treating the general problem $\mathbf{P}\left(\mathbf{D} ; k_{0}\right)$, $k_{0}>0$, it seems expedient to consider first the zero-frequency case.

The zero-frequency case. In the low-frequency limit $k_{0} \rightarrow 0$, the functions $\phi_{m}\left(\theta ; k_{0}\right)$ and $\mathbf{V}\left(\theta ; k_{0}\right)$ admit an asymptotic expansion of the form

$$
\phi_{m}\left(\theta ; k_{0}\right)=\phi_{m}(\theta ; 0)+ \begin{cases}O\left(k_{0} \ln k_{0}\right), & m=0,1, \\ O\left(k_{0}\right), & m=2,3, \ldots\end{cases}
$$


and

$$
\mathbf{V}\left(\theta ; k_{0}\right)=\mathbf{V}(\theta ; 0)+ \begin{cases}0, & \text { for the radiation problem, } \\ O\left(k_{0}\right), & \text { for the diffraction problem }\end{cases}
$$

where

$$
\begin{gathered}
\phi_{0}(\theta ; 0)=-j \operatorname{Im}_{i}\left\{w^{\prime}(\theta) /(\pi w(\theta))\right\}, \quad \phi_{1}(\theta ; 0)=-\operatorname{Im}_{i}\left\{w^{\prime}(\theta) /\left(\pi w^{2}(\theta)\right)\right\} \\
\phi_{m}(\theta ; 0)=\cos (m \theta), \quad m=2,3, \ldots
\end{gathered}
$$

and

$\mathbf{V}(\theta ; 0)= \begin{cases}\xi_{2} x_{3}^{\prime}(\theta)-\xi_{3} x_{2}^{\prime}(\theta)-\xi_{4} x_{4}^{\prime}(\theta), & \text { for the radiation problem, } \\ A\left(x_{2}^{\prime}(\theta)+j x_{3}^{\prime}(\theta)\right), & \text { for the diffraction problem, }\end{cases}$

with

$$
x_{4}(\theta)=\left(x_{2}^{2}(\theta)+x_{3}^{2}(\theta)\right) / 2
$$

Clearly,

$$
\lim _{k_{0} \rightarrow 0} \phi_{m}\left(\theta ; k_{0}\right)=\phi_{m}(\theta ; 0), \quad m \geq 0, \quad \text { and } \quad \lim _{k_{0} \rightarrow 0} \mathbf{V}\left(\theta ; k_{0}\right)=\mathbf{V}(\theta ; 0),
$$

and the functions $\mathbf{V}(\theta ; 0), \phi_{m}(\theta ; 0), m \geq 0$, belong to $L^{p}(-\pi, 0)$ (cf. Lemma 3.1).

The above asymptotic expansions permit us to consider the zero-frequency problem (formal limit problem) $\mathbf{P}(\mathbf{D} ; 0)$. This simple problem has been extensively studied by many authors since it provides useful hydrodynamic quantities for floating cylinders. See, e.g., [4] for a recent survey. However, the relation of its solution with the solution of the frequency-dependent problem $\mathbf{P}\left(\mathbf{D} ; k_{0}\right)$ as $k_{0} \rightarrow 0$, is by no means trivial, and it can be found in [39, 41] (for smooth, symmetric bodies), and in [4, 5] (for the general case).

We shall now establish the solvability and the regularity results for $\mathbf{P}(\mathbf{D} ; 0)$, working along the same lines as for the general problem $\mathbf{P}\left(\mathbf{D} ; k_{0}\right)$. This simple case is free of obscured technicalities and permits us to present clearly the underlying ideas. ${ }^{1}$

Theorem 3.2. Let $\partial D_{B} \in W^{1, p}, 1<p<\infty$, Then, the sequence $\left\{\phi_{m}(\theta ; 0)\right\}_{0}^{\infty}$ forms a Schauder basis of $L^{p}(-\pi, 0)$.

Proof. Motivated by the fact that $\phi_{m}(\theta ; 0)=\cos (m \theta), m \geq 2$, we decompose the space $L^{p}=L^{p}(-\pi, 0)$ in the form $L^{p}=L_{A}^{p} \oplus L_{B}^{p}$, where $L_{A}^{p}=[1, \cos \theta]$ and $L_{B}^{p}=$ $[\cos (m \theta), m \geq 2]$. It should be noted that this decomposition is introduced here, although it could be avoided as regards the formal limit problem, since it becomes necessary in the treatment of the general case $k_{0}>0$. See Theorem 4.3.

Let $v(\theta)$ be an arbitrary element of $L^{p}$, and consider the element

$$
u(\theta)=v(\theta)-a_{0} \phi_{0}(\theta ; 0)-a_{1} \phi_{1}(\theta ; 0),
$$

where the coefficients $a_{n}, n=0,1$, are determined so that $u(\theta)$ belongs to $L_{B}^{p}$. This is equivalent to

$$
\tilde{u}_{c}(n)=0, \quad n=0,1,
$$

${ }^{1}$ We are grateful to the referee of this paper who recommended the separate treatment of the zero-frequency case. 
where $\tilde{u}_{c}(n)$ is the $n$ th-order cosine-Fourier coefficient of the function $u(\theta)$ defined by

$$
\tilde{u}_{c}(n)=\frac{1}{\pi} \int_{-\pi}^{0} u(\theta) \cos (n \theta) d \theta, \quad n=0,1,2, \ldots
$$

Condition (3.26) can be written as

$$
a_{0} \tilde{\phi}_{0 c}(n ; 0)+a_{1} \tilde{\phi}_{1 c}(n ; 0)=\tilde{v}_{c}(n), \quad n=0,1,
$$

which constitutes a linear system for the coefficients $a_{n}, n=0,1$. This system is always solvable, since its determinant $\Delta(0)$, can be expressed in the form

$$
\Delta(0)=\frac{j}{\pi^{3}} \int_{-\pi}^{0} \frac{x_{3}(\theta)}{|w(\theta)|^{2}} \sin \theta d \theta
$$

which is always a positive quantity.

Thus, given any $v(\theta) \in L^{p}$, we can construct a unique $u(\theta) \in L_{B}^{p}$, and using the basis property of the trigonometric sequence $\{\cos (m \theta)\}_{2}^{\infty}$ in $L_{B}^{p}$, we obtain the expansion

$$
u(\theta)=\sum_{m=2}^{\infty} \tilde{u}_{c}(m) \cos (m \theta), \quad \text { in } L_{B}^{p} .
$$

Note that the latter series converges in the $L_{B}^{p}$ norm (see [20, p. 50]). Relation (3.30), in conjunction with $(3.25)$, provides us with the expansion

$$
v(\theta)=\sum_{m=0}^{\infty} f_{m}(v) \phi_{m}(\theta ; 0), \quad \text { in } L^{p},
$$

where,

$$
f_{m}(v)=a_{m}, \quad m=0,1, \quad \text { and } \quad f_{m}(v)=\tilde{u}_{c}(m), \quad m \geq 2 .
$$

The latter relation shows that any element $v(\theta)$ of $L^{p}$ can be expanded in terms of the functions $\phi_{m}(\theta, 0), m \geq 0$, and the corresponding series converges in the $L^{P}$ norm. This means that the sequence $\left\{\phi_{m}(\theta ; 0)\right\}_{0}^{\infty}$ forms a Schauder basis of $L^{p}(-\pi, 0)$, and completes the proof of the theorem.

In what follows it is very important to know the behaviour of the sequence of the expansion coefficients $\left\{f_{m}(v)\right\}_{0}^{\infty}$ with respect to $m$. The basic tool to obtain this information is the Hausdorff-Young Theorem [20, p. 98], which states that the sequence of the Fourier coefficients $\left\{\tilde{u}_{c}(m)\right\}_{2}^{\infty}$, defined through the expansion (3.30), belongs to the sequence space $\ell^{q^{*}}$, for $q^{*}=\max \{2, p /(p-1)\}$. This fact, in conjunction with relation (3.32), leads to the estimate

$$
\sum_{m=0}^{\infty}\left|f_{m}(v)\right|^{q^{*}}<\infty, \quad q^{*}=\max \{2, p /(p-1)\} .
$$

Theorem 3.2 in conjunction with relation (3.33) immediately implies that, for a given $\partial D_{B} \in W^{1, p}, p>1$, the formal limit problem $\mathbf{P}(\mathbf{D} ; 0)$ admits a unique solution $\left\{D_{m}(0)\right\}_{0}^{\infty}$ in the sequence space $\ell^{q^{*}}, q^{*}=\max \{2, p /(p-1)\}$. We shall now show that the estimate $\left\{D_{m}(0)\right\}_{0}^{\infty} \in \ell^{q^{*}}$, is the best possible in the case where the only fact that 
we know about the body boundary is that it belongs to the class $W^{1, p}$. (Stronger results will be subsequently established for piecewise smooth boundaries.) Taking into account relations (3.20)-(3.23), we can write the body-boundary condition (3.14), for the case $k_{0}=0$, in the form

$$
D_{0}(0) \phi_{0}(\theta ; 0)+D_{1}(0) \phi_{1}(\theta ; 0)+\sum_{m=2}^{\infty} D_{m}(0) \cos (m \theta)=\mathbf{V}(\theta ; 0) \text {. }
$$

Then, taking the cosine-Fourier coefficients of the two members of (3.34), we obtain the following relations for the expansion coefficients $D_{m}(0)$ :

$$
\begin{gathered}
D_{0}(0) \tilde{\phi}_{0 c}(m ; 0)+D_{1}(0) \tilde{\phi}_{1 c}(m ; 0)=\tilde{\mathbf{V}}_{c}(m ; 0), \quad m=0,1, \\
D_{0}(0) \tilde{\phi}_{0 c}(m ; 0)+D_{1}(0) \tilde{\phi}_{1 c}(m ; 0)+D_{m}(0) / 2=\tilde{\mathbf{V}}_{c}(m ; 0), \quad m \geq 2 .
\end{gathered}
$$

These equations show that the order $O\left(D_{m}(0)\right)$ is dominated by $O\left(\widetilde{\mathbf{V}}_{c}(m ; 0)\right)$, which implies that the estimate $\left\{D_{m}(0)\right\}_{0}^{\infty} \in \ell^{q^{*}}$ cannot, in general, be improved, since the estimate $\left\{\widetilde{\mathbf{V}}_{c}(m ; 0)\right\}_{0}^{\infty} \in \ell^{q^{*}}$ is the best possible. Indeed, there exist even continuous functions $\mathbf{V}(\theta ; 0)$ (e.g., continuous $\left.x_{k}^{\prime}(\theta), k=2,3,4\right)$ with no better estimates for their Fourier coefficients $\widetilde{\mathbf{V}}_{c}(m ; 0)$; See, e.g., [8, p. 228].

To obtain individual estimates ${ }^{2}$ for the coefficients $D_{m}(0)$, the body boundaries should be confined to an appropriate subclass of $W^{1, p}$. For the subclass consisting of polygonal body boundaries we can prove the following:

TheOREM 3.3. Let $\partial D_{B}=\partial D_{B}^{-} \cup \partial D_{B}^{+}$be a polygonal section of which the smaller exterior angle is $\alpha \pi, 0<\alpha<2, \alpha \neq 1$. Then, $D_{m}(0)=O\left(m^{-\alpha}\right)$, and this estimate is the best possible.

We shall sketch here a proof of the theorem, referring to Sec. 6, Theorem 6.1 for a complete proof.

Sketch of the Proof. Using the explicit form of the conformal mapping function $f(\zeta)$ (obtained via the Schwartz-Christoffel transformation), we can express the Fourier coefficients of $w^{\prime}(\theta)$ in terms of the incomplete Gamma function. Then, applying standard asymptotic results for this function, in conjunction with some geometric relations given in Appendix $\mathrm{A}$ and Theorem $\mathrm{C} 1$ of Appendix $\mathrm{C}$ we can establish the estimates,

$$
\tilde{\mathbf{V}}_{c}(m ; 0)=O\left(m^{-\alpha}\right), \quad \tilde{\phi}_{0 c}(m ; 0)=O\left(m^{-\alpha}\right), \quad \tilde{\phi}_{1 c}(m ; 0)=O\left(m^{-\alpha}\right) .
$$

In the course of the proof it becomes clear that the above estimates are the best possible. These estimates, in conjunction with relation $(3.35 \mathrm{~b})$ give us the individual estimates for the coefficients $D_{m}(0)$ stated in the theorem.

Proceeding along the same lines as in the above theorem, we can prove that the estimate given in Theorem 3.3 remains valid for piecewise smooth boundaries $\partial D_{B}$, consisting of sufficiently smooth arcs (curvilinear polygons). See Remark 6.1.

When the body boundary is sufficiently smooth (see Definition 6.1), we obtain $D_{m}(0)=O\left(m^{-2}\right)$. Note that, in the case of heave and roll motion, this estimate

\footnotetext{
${ }^{2}$ We shall call an individual estimate of the sequence $\left\{D_{m}\right\}_{0}^{\infty}$ any relation of the form $D_{m}=O(\lambda(m))$, where $\lambda(m)$ is a monotone sequence.
} 
cannot be further improved however smooth the boundary is, while in the case of sway motion stronger results can be obtained depending on the smoothness of the body boundary $\partial D_{B}$. See [4, Appendix 3].

Motivated by the basis property of the sequence $\left\{\phi_{m}(\theta ; 0)\right\}_{0}^{\infty}$ proved in Theorem 3.2 , we shall prove in the next section that the sequence $\left\{\phi_{m}\left(\theta ; k_{0}\right)\right\}_{0}^{\infty}, k_{0}>0$, is also a basis of $L^{p}(-\pi, 0), 1<p \leq 2$, for all $k_{0} \in \mathbb{R}^{+}-F$, where $F$ is a set of isolated values of $k_{0}$ (possibly empty).

4. The basis property of $\phi_{m}\left(\theta ; k_{0}\right), m=0,1, \ldots$. To prove the basis property of the sequence $\left\{\phi_{m}\left(\theta ; k_{0}\right)\right\}_{0}^{\infty}$ use is made of the theory of bases in Banach spaces (see, e.g., [34]) and the spectral properties of analytic families of compact operators [13, $17,35]$. We recall here some basic notions and results, which will be extensively used in this section.

Definition 4.1 (Singer [34, p. 68]). Two sequences (or bases) $\left\{x_{n}\right\}_{0}^{\infty}$ and $\left\{y_{n}\right\}_{0}^{\infty}$ in a Banach space $E$ are said to be equivalent, if there exists a bounded invertible operator (automorphism) $T: E \rightarrow E$, such that $T x_{n}=y_{n}$, for every $n$.

Definition 4.2. Let $\left\{x_{n}\right\}_{0}^{\infty}$ be a basis of a Banach space $E$ and $\left\{f_{n}\right\}_{0}^{\infty} c E^{\prime}$ (the dual of $E$ ) be its associated sequence of coefficient functionals (a.s.c.f.). We shall say that the pair $\left(E,\left\{x_{n}\right\}_{0}^{\infty}\right)$ possesses $\ell^{p}$-bounded a.s.c.f. if there exists a positive constant $A$ such that

$$
\left\|f_{n}(x)\right\|_{\ell^{p}} \equiv\left(\sum_{n=0}^{\infty}\left|f_{n}(x)\right|^{p}\right)^{1 / p} \leq A\|x\|_{E}, \quad \text { for all } x \in E .
$$

LEMMA 4.1. (i) If a sequence $\left\{y_{n}\right\}_{0}^{\infty}$ in a Banach space $E$ is equivalent to a (Schauder, Riesz) basis $\left\{x_{n}\right\}_{0}^{\infty}$ of $E$, then it is also a (Schauder, Riesz) basis of $E$.

(ii) If, moreover, the pair $\left(E,\left\{x_{n}\right\}_{0}^{\infty}\right)$ possesses $\ell^{p}$-bounded a.s.c.f., then the pair $\left(E,\left\{y_{n}\right\}_{0}^{\infty}\right)$ also possesses $\ell^{p}$-bounded a.s.c.f.

Let us now examine the basis property of the system

$$
\left\{\psi_{m}\left(\theta ; k_{0}\right)\right\}_{0}^{\infty}=\left\{1, \cos \theta, \phi_{m}\left(\theta ; k_{0}\right)=\cos (m \theta)+k_{0} g_{m}(\theta) / m, m=2,3, \ldots\right\},
$$

which is obtained by $\left\{\phi_{m}\left(\theta ; k_{0}\right)\right\}_{0}^{\infty}$ if we replace $\phi_{0}\left(\theta ; k_{0}\right)$ and $\phi_{1}\left(\theta ; k_{0}\right)$ by the simpler functions 1 and $\cos \theta$, respectively.

Theorem 4.1. Let $\partial D_{B} \in W^{1, p}, 1<p \leq \infty$, and $k_{0} \in \mathbb{C}_{j}$. Then, for sufficiently small $\left|k_{0}\right|$ we have

(i) If $1<p \leq 2$, the sequence $\left\{\psi_{m}\left(\theta ; k_{0}\right)\right\}_{0}^{\infty}$ is a Schauder basis of $L^{p}(-\pi, 0)$ with $\ell^{q}$-bounded a.s.c.f., $q=p /(p-1)$;

(ii) If $2 \leq p \leq \infty$, the sequence $\left\{\psi_{m}\left(\theta ; k_{0}\right)\right\}_{0}^{\infty}$ is a (nonorthogonal) Riesz basis of $L^{2}(-\pi, 0)$.

When $p \neq \infty$ the above results are the best possible (see Remark 3.1).

Proof. (i) First we note that the trigonometric system $\{\cos (m \theta)\}_{0}^{\infty}$ is a Schauder basis of $L^{p}=L^{p}(-\pi, 0), 1<p<\infty$, and, in virtue of the Hausdorff-Young Theorem [20, p. 98], the pair $\left(L^{p},\{\cos (m \theta)\}_{0}^{\infty}\right), 1<p \leq 2$, possesses $\ell^{q}$-bounded a.s.c.f. $q=p /(p-1)$. That is

$$
\left\|f_{m}(x)\right\|_{\ell q} \leq A\|x\|_{L^{p}}, \quad \text { for all } x \in L^{p}(-\pi, 0), \quad 1<p \leq 2,
$$


where $A>0$, and $\left\{f_{m}(x)\right\}_{0}^{\infty}$ are the a.s.c.f. given by

$$
f_{m}(x)=\frac{1}{\pi} \int_{\pi}^{0} x(\theta) \cos (m \theta) d \theta, \quad m=0,1, \ldots
$$

We now define the operator $T_{k_{0}}, k_{0} \in \mathbb{C}_{j}$, by setting

$$
T_{k_{0}} x=2 \sum_{m=0}^{\infty} f_{m}(x)\left(\cos (m \theta)-\psi_{m}\left(\theta ; k_{0}\right)\right)
$$

This operator is defined on $L^{p}$, its range lies in $L^{p}$ and it is bounded. For any given $x \in L^{p}$, we can apply the Hölder inequality obtaining

$$
\begin{aligned}
& \sum_{m=0}^{\infty}\left|f_{m}(x)\right|\left\|\cos (m \theta)-\psi_{m}\left(\theta ; k_{0}\right)\right\|_{L^{p}} \\
& \quad \leq\left(\sum_{m=0}^{\infty}\left|f_{m}(x)\right|^{q}\right)^{1 / q}\left(\sum_{m=0}^{\infty}\left\|\cos (m \theta)-\psi_{m}\left(\theta ; k_{0}\right)\right\|_{L^{p}}^{p}\right)^{1 / p},
\end{aligned}
$$

which, in conjunction with (3.19) and (4.3) leads to

$$
\left\|T_{k_{0}} x\right\|_{L^{p}} \leq 2 A\left|k_{0}\right|\left\|w^{\prime}(\theta)\right\|_{L^{p}}\left(\sum_{m=2}^{\infty} m^{-p}\right)^{1 / p}\|x\|_{L^{p}}=B\left|k_{0}\right|\|x\|_{L^{p}} .
$$

The latter inequality proves the boundedness of $T_{k_{0}}: L^{p} \rightarrow L^{p}$, and that $\left\|T_{k_{0}}\right\| \leq B\left|k_{0}\right|$. But then, for $\left|k_{0}\right|<B^{-1},\left\|T_{k_{0}}\right\|<1$, and thus, the operator $I-T_{k_{0}}$ is bounded and invertible on $L^{p}$. Now, since $\left(I-T_{k_{0}}\right)(\cos (m \theta))=\psi_{m}\left(\theta ; k_{0}\right), m=0,1, \ldots$, Lemma 4.1 permits us to conclude that the sequence $\left\{\psi_{m}\left(\theta ; k_{0}\right)\right\}_{0}^{\infty}$ is a basis of $L^{p}, 1<p \leq 2$, equivalent to $\{\cos (m \theta)\}_{0}^{\infty}$, and that the pair $\left(L^{p},\left\{\psi_{m}\left(\theta ; k_{0}\right)\right\}_{0}^{\infty}\right), 1<p \leq 2$, possesses $\ell^{q}$-bounded a.s.c.f., for $\left|k_{0}\right|<B^{-1}$.

(ii) For the case $p=2$ the trigonometric system $\{\cos (m \theta)\}_{0}^{\infty}$ is an orthonormal (thus a Riesz) basis of $L^{2}$, and, consequently $\left\{\psi_{m}\left(\theta ; k_{0}\right)\right\}_{0}^{\infty}$ is a (nonorthogonal) Riesz basis of $L^{2}$, for $\left|k_{0}\right|<B^{-1}$. This result cannot be, in general, extended for $p>2$, since the Hausdorff-Young Theorem cannot be extended to $p>2$ [8, p. 223]. Thus, for $p \geq 2$ we should content ourselves with the $L^{2}$-basis property of $\left\{\psi_{m}\left(\theta, k_{0}\right)\right\}_{0}^{\infty}$. This completes the proof of the Theorem.

The basis property of $\left\{\psi_{m}\left(\theta ; k_{0}\right)\right\}_{0}^{\infty}$ would be extended for $\left|k_{0}\right| \geq B^{-1}, k_{0} \in \mathbb{C}_{j}$, if it would be possible to extend there the operator $I-T_{k_{0}}$ as an automorphism on $L^{p}$. We shall now prove that such an extension is indeed possible for almost all $k_{0} \in \mathbb{C}_{j}$. To proceed to this direction we need the following two properties of the operator $T_{k_{0}}$.

LEMMA 4.2. The operator $T_{k_{0}}$ is compact for each $k_{0} \in \mathbb{C}_{j}$, and analytic with respect to $k_{0} \in \mathbb{C}_{j}$.

Proof. We define the finite-dimensional operater $T_{k_{0}}^{N}, k_{0} \in \mathbb{C}_{j}$, by

$$
T_{k_{0}}^{N} x=\sum_{m=0}^{N} f_{m}(x)\left(\cos (m \theta)-\psi_{m}\left(\theta ; k_{0}\right)\right) .
$$


Then, in virtue of (3.17) and (4.3) we have

$$
\left\|T_{k_{0}}^{N}-T_{k_{0}}\right\| \leq A\left|k_{0}\right|\left\|w^{\prime}(\theta)\right\|_{L^{p}}\left(\sum_{m=N+1}^{\infty} m^{-p}\right)^{1 / p},
$$

which implies that $\left\|T_{k_{0}}^{N}-T_{k_{0}}\right\| \rightarrow 0$, as $N \rightarrow \infty$, from which the compactness of the operator $T_{k_{0}}$ follows immediately.

The analyticity of $T_{k_{0}}$ follows at once by making use of the fact that, for each $x \in L^{p}$ and $f^{\prime} \in L^{q}=\left(L^{p}\right)^{\prime}, q=p /(p-1)$, the function

$$
h\left(k_{0}\right)=f^{\prime}\left(T_{k_{0}} x\right)=\sum_{m=0}^{\infty} f_{m}(x) f^{\prime}\left(\cos (m \theta)-\psi_{m}\left(\theta ; k_{0}\right)\right),
$$

is well defined and analytic with respect to $k_{0} \in \mathbb{C}_{j}[36$, p. 205].

The extension of $I-T_{k_{0}}$ as an automorphism on $L^{p}$ for $k_{0} \in \mathbb{C}_{j}-S$, where $S$ is a set of isolated points, is now a direct consequence of Theorem 4.1 and a theorem of Gohberg on the invertibility of $I-T_{k_{0}}$ when $T_{k_{0}}$ is analytic [13, p. 21]. Thus, we have proved the following:

Theorem 4.2. Let $\partial D_{B} \in W^{1, p}, 1<p \leq \infty$, and $k_{0} \in \mathbb{C}_{j}$. Then, for all $k_{0} \in \mathbb{C}_{j}-S$, where $S$ is a set of isolated points, the conclusions (i) and (ii) of Theorem 4.1 remain valid.

Unfortunately, the above results cannot be directly transferred to the system $\left\{\phi_{m}\left(\theta ; k_{0}\right)\right\}_{0}^{\infty}$. The reason is that $\phi_{0}\left(\theta ; k_{0}\right)$ and $\phi_{1}\left(\theta ; k_{0}\right)$ are not "small" perturbations of 1 and $\cos \theta$, respectively, and thus we cannot ensure that $\left\|T_{k_{0}}\right\|<1$ for small $\left|k_{0}\right|$. To cope with this difficulty we shall decompose the space $L^{p}$ into a direct sum of two subspaces, and we shall proceed in a manner analogous to that used in Theorem 3.2.

THEOREM 4.3. Let $\partial D_{B} \in W^{1, p}, 1<p \leq \infty$, and let $q=p /(p-1)$ be the conjugate exponent. Let also $Q \subset \mathbb{C}_{j}-\{0\}$ be an open bounded simply-connected region, containing an interval of the form $(0, h), h>0$.

Then, for all $k_{0} \in Q-S$, where $S$ is a set of arbitrarily small plane measure, we have

(i) If $1<p \leq 2$, the sequence $\left\{\phi_{m}\left(\theta ; k_{0}\right)\right\}_{0}^{\infty}$ is a Schauder basis of $L^{p}(-\pi, 0)$ with $\ell^{q}$-bounded a.s.c.f;

(ii) If $2 \leq p \leq \infty$, the sequence $\left\{\phi_{m}\left(\theta ; k_{0}\right)\right\}_{0}^{\infty}$ is a (nonorthogonal) Riesz basis of $L^{2}(-\pi, 0)$.

When $p \neq \infty$ the above results are the best possible (see Remark 3.1). When $p=$ $\infty$ stronger results would be obtained, dependent on the smoothness of the body boundary $\partial D_{B}$.

Since the proof is lengthy and cumbersome we first give a description of its basic steps.

Step One: We decompose the space $L^{p}(-\pi, 0)$ into two subspaces $L_{A}^{p}$ and $L_{B}^{p}$ as in Theorem 3.2, and we appropriately modify the multipoles $\phi_{m}\left(\theta ; k_{0}\right), m \geq 2$, so that the modified ones $\phi_{m}^{*}\left(\theta ; k_{0}\right)$ belong to the subspace $L_{B}^{p}$.

Step Two: We prove that the sequence $\left\{\phi_{m}^{*}\left(\theta ; k_{0}\right)\right\}_{2}^{\infty}$ forms a basis of $L_{B}^{p}$ equivalent to the trigonometric basis $\{\cos (m \theta)\}_{2}^{\infty}$. 
Step Three: We prove that the set of all multipoles $\phi_{m}\left(\theta ; k_{0}\right), m \geq 0$, forms a basis of the whole space $L^{p}$.

Step Four: We prove that the pair $\left(L^{p},\left\{\phi_{m}\left(\theta ; k_{0}\right)\right\}_{0}^{\infty}\right)$ possesses $\ell^{q}$-bounded a.s.c.f.

Proof. (i) The case $1<p \leq 2$.

Step One: We decompose the space $L^{p}=L^{p}(-\pi, 0)$ in the form $L^{p}=L_{A}^{p} \oplus L_{B}^{p}$, where $L_{A}^{p}=[1, \cos \theta]$ and $L_{B}^{p}=[\cos (m \theta), m \geq 2]$, and we modify the elements $\phi_{m}\left(\theta ; k_{0}\right), m \geq 2$, in such a manner that the modified ones become "small" perturbations of $\cos (m \theta), m \geq 2$, belonging to $L_{B}^{p}$.

To start, we observe that the sequence $\{\cos (m \theta)\}_{2}^{\infty}$ is a basis of $L_{B}^{p}$ and that, in virtue of the Hausdorff-Young Theorem, the pair $\left(L^{p},\{\cos (m \theta)\}_{2}^{\infty}\right), 1<p \leq 2$, possesses $\ell^{q}$-bounded a.s.c.f., that is

$$
\left(\sum_{m=2}^{\infty}\left|f_{m}(x)\right|^{q}\right)^{1 / q} \leq A\|x\|_{L_{B}^{p}}, \quad \text { for all } x \in L_{B}^{p},
$$

where $\left\{f_{m}(x)\right\}_{2}^{\infty}$ are the a.s.c.f. defined by (4.3).

Since, in general, $\phi_{m}\left(\theta ; k_{0}\right), m \geq 2$, do not belong to $L_{B}^{p}$ (they have components in $L_{A}^{p}$ too), we cannot deduce any basis property of the sequence $\left\{\phi_{m}\left(\theta ; k_{0}\right)\right\}_{2}^{\infty}$ in $L_{B}^{p}$. In this connection we define

$$
\phi_{m}^{*}\left(\theta ; k_{0}\right)=\phi_{m}\left(\theta ; k_{0}\right)-a_{m 0} \phi_{0}\left(\theta ; k_{0}\right)-a_{m 1} \phi_{1}\left(\theta ; k_{0}\right), \quad m \geq 2,
$$

where $a_{m n} \in \mathbb{C}_{j}, m \geq 2, n=0,1$. Then, $\phi_{m}^{*}\left(\theta ; k_{0}\right) \in L_{B}^{p}, m \geq 2$, if and only if $f_{n}\left(\phi_{m}^{*}\left(\theta ; k_{0}\right)\right)=0, n=0,1, m \geq 2$. This condition leads to the following linear system for the coefficients $a_{m n}$

$$
\begin{aligned}
& a_{m 0} f_{0}\left(\phi_{0}\left(\theta ; k_{0}\right)\right)+a_{m 1} f_{0}\left(\phi_{1}\left(\theta ; k_{0}\right)\right)=k_{0} f_{0}\left(g_{m}(\theta)\right) / m, \\
& a_{m 0} f_{1}\left(\phi_{0}\left(\theta ; k_{0}\right)\right)+a_{m 1} f_{1}\left(\phi_{1}\left(\theta ; k_{0}\right)\right)=k_{0} f_{1}\left(g_{m}(\theta)\right) / m,
\end{aligned}
$$

which is uniquely solvable, for each $m \geq 2$, provided that

$$
\Delta\left(k_{0}\right) \equiv f_{0}\left(\phi_{0}\left(\theta ; k_{0}\right)\right) f_{1}\left(\phi_{1}\left(\theta ; k_{0}\right)\right)-f_{0}\left(\phi_{1}\left(\theta ; k_{0}\right)\right) f_{1}\left(\phi_{0}(\theta ; k 0)\right) \neq 0 .
$$

The function $\Delta\left(k_{0}\right)$ is analytic with respect to $k_{0} \in Q$, and it admits of the following asymptotic approximation for small $k_{0}[19]$

$$
\Delta\left(k_{0}\right)=\Delta(0)+O\left(k_{0} \ln k_{0}\right),
$$

where $\Delta(0)$ is given by (3.19). Equation (4.13) implies that $\Delta\left(k_{0}\right)$ cannot be identically equal to zero; it may have only isolated zeros in $Q$. If this is the case we modify the region $Q$ by excluding from it arbitrarily small neighborhoods of the zeros of $\Delta\left(k_{0}\right)$ and introducing cuts, so that the modified region, $Q_{1}=Q-S_{1}$ is simply-connected. Then, for $k_{0} \in Q_{1}$, we have $\Delta\left(k_{0}\right) \neq 0$, and the system (4.12) is always solvable there.

The solution of the system (4.12) can be written in the form

$$
a_{m n}=a_{m n}\left(k_{0}\right)=k_{0} b_{m n}\left(k_{0}\right) / m, \quad n=0,1, m \geq 2,
$$

with

$$
\left|b_{m n}\left(k_{0}\right)\right|<B_{n},
$$


where $B_{n}=B_{n}\left(Q_{1}\right)$ is independent of $m$ and $k_{0} \in Q_{1}$. Note that $a_{m n}\left(k_{0}\right)$ and $b_{m n}\left(k_{0}\right)$, $m \geq 2, n=0,1$, are analytic functions of $k_{0} \in Q_{1}$. Now taking into account (3.17), (4.11), and (4.14), we see that the modified elements $\phi_{m}^{*}\left(\theta ; k_{0}\right) \in L_{B}^{p}, m \geq 2$, may be written in the form

$$
\phi_{m}^{*}\left(\theta ; k_{0}\right)=\cos (m \theta)+k_{0} g_{m}^{*}\left(\theta ; k_{0}\right) / m, \quad m \geq 2,
$$

where

$$
g_{m}^{*}\left(\theta ; k_{0}\right)=g_{m}(\theta)-b_{m 0}\left(k_{0}\right) \phi_{0}\left(\theta ; k_{0}\right)-b_{m 1}\left(k_{0}\right) \phi_{1}\left(\theta ; k_{0}\right) .
$$

Step Two: Now, we shall show that $\left\{\phi_{m}^{*}\left(\theta ; k_{0}\right)\right\}_{2}^{\infty}$ forms a basis of $L_{B}^{p}$, equivalent to $\{\cos (m \theta)\}_{2}^{\infty}$. The proof proceeds along the lines of proofs of Theorems 4.1 and 4.2. Define the operator $T_{k_{0}}$ on $L_{B}^{p}$ by

$$
T_{k_{0}} x=2 \sum_{m=2}^{\infty} f_{m}(x)\left(\cos (m \theta)-\phi_{m}^{*}\left(\theta ; k_{0}\right)\right), \quad k_{0} \in Q_{1} .
$$

In virtue of (4.10), (4.15), and (4.16) we can see that the operator $T_{k_{0}}, k_{0} \in Q_{1}$, is compact (cf. Lemma 4.2), and moreover that

$$
\left\|T_{k_{0}}\right\| \leq 6 A\left|k_{0}\right|\left(\left\|w^{\prime}(\theta)\right\|_{L^{p}}^{p}+B_{0}^{p}\left(Q_{1}\right) M_{0}^{p}\left(Q_{1}\right)+B_{1}^{p}\left(Q_{1}\right) M_{1}^{p}\left(Q_{1}\right)\right)^{1 / p}\left(\sum_{m=2}^{\infty} m^{-p}\right)^{1 / p},
$$

where $M_{n}\left(Q_{1}\right)=\sup \left\{\left\|\phi_{n}\left(\theta ; k_{0}\right)\right\|_{L^{p}}, k_{0} \in Q_{1}\right\}<\infty, n=0,1$. Thus, for sufficiently small $\left|k_{0}\right|$, we obtain that $\left\|T_{k_{0}}\right\|<1$. On the other hand, since the operator $T_{k_{0}}$ is analytic with respect to $k_{0} \in Q_{1}$ (cf. Lemma 4.2), we can use Theorem 5.1 of Gohberg $\left[13\right.$, p. 21] to deduce the invertibility of the operator $I-T_{k_{0}}$ for all $k_{0} \in Q_{1}-S_{2}$, where $S_{2}$ is a set of isolated points of $k_{0}$. This fact, in conjunction with the relation $\left(I-T_{k_{0}}\right)(\cos (m \theta))=\phi_{m}^{*}\left(\theta ; k_{0}\right), m \geq 2$, and Lemma 4.1, implies that $\left\{\phi_{m}^{*}\left(\theta ; k_{0}\right)\right\}_{2}^{\infty}$ is a basis of $L_{B}^{p}$, equivalent to $\{\cos (m \theta)\}_{2}^{\infty}$, and that the pair $\left(L_{B}^{p},\left\{\phi_{m}^{*}\left(\theta ; k_{0}\right)\right\}_{2}^{\infty}\right)$ possesses $\ell^{q}$-bounded a.s.c.f. for all $k_{0} \in Q_{2}=Q_{1}-S_{2}=Q-S$, where $S=S_{1} \cup S_{2}$ is a set of arbitrarily small plane measure.

Step Three: We now proceed to show that $\left\{\phi_{m}\left(\theta ; k_{0}\right)\right\}_{0}^{\infty}$ forms a basis of $L^{p}$ for all $k_{0} \in Q_{2}$. Let $v\left(\theta ; k_{0}\right) \in L^{p}$ and consider the element

$$
u\left(\theta ; k_{0}\right)=v\left(\theta ; k_{0}\right)-a_{0}\left(k_{0}\right) \phi_{0}\left(\theta ; k_{0}\right)-a_{1}\left(k_{0}\right) \phi_{1}\left(\theta ; k_{0}\right),
$$

where $a_{n}\left(k_{0}\right), n=0,1$, is the solution of the linear system

$$
\begin{aligned}
& a_{0}\left(k_{0}\right) f_{0}\left(\phi_{0}\left(\theta ; k_{0}\right)\right)+a_{1}\left(k_{0}\right) f_{0}\left(\phi_{1}\left(\theta ; k_{0}\right)\right)=f_{0}\left(v\left(\theta ; k_{0}\right)\right), \\
& a_{0}\left(k_{0}\right) f_{1}\left(\phi_{0}\left(\theta ; k_{0}\right)\right)+a_{1}\left(k_{0}\right) f_{1}\left(\phi_{1}\left(\theta ; k_{0}\right)\right)=f_{1}\left(v\left(\theta ; k_{0}\right)\right) .
\end{aligned}
$$

Then, clearly, $f_{n}\left(u\left(\theta ; k_{0}\right)\right)=0, n=0,1$, which means that $u\left(\theta ; k_{0}\right) \in L_{B}^{p}$, and consequently we may write

$$
u\left(\theta ; k_{0}\right)=\sum_{m=2}^{\infty} h_{m}^{*}\left(u ; k_{0}\right) \phi_{m}^{*}\left(\theta ; k_{0}\right)
$$

with

$$
\left(\sum_{m=2}^{\infty}\left|h_{m}^{*}\left(u ; k_{0}\right)\right|^{q}\right)^{1 / q} \leq A_{1}\left(Q_{2}\right)\|u\|_{L_{B}^{p}}
$$


Now combining (4.11), (4.19), and (4.21), we obtain

$$
\begin{aligned}
v\left(\theta ; k_{0}\right) & =\sum_{m=0}^{1} a_{m}\left(k_{0}\right) \phi_{m}\left(\theta ; k_{0}\right)+\sum_{m=2}^{\infty} h_{m}^{*}\left(u ; k_{0}\right) \phi_{m}^{*}\left(\theta ; k_{0}\right) \\
& =\sum_{m=0}^{\infty} h_{m}\left(v ; k_{0}\right) \phi_{m}\left(\theta ; k_{0}\right)
\end{aligned}
$$

where

$$
\begin{aligned}
h_{n}\left(v ; k_{0}\right)= & a_{n}\left(k_{0}\right)-\sum_{m=2}^{\infty} h_{m}^{*}\left(u ; k_{0}\right) a_{m n}\left(k_{0}\right), \quad n=0,1, \\
& h_{n}\left(v ; k_{0}\right)=h_{n}^{*}\left(u ; k_{0}\right), \quad n \geq 2 .
\end{aligned}
$$

Equation (4.23), in conjunction with (4.15), (4.22), and (4.24), shows that each element $v\left(\theta ; k_{0}\right) \in L^{p}$ has a unique expansion in terms of $\phi_{m}\left(\theta ; k_{0}\right), m=0,1,2, \ldots$, convergent in the norm. Thus, the system $\left\{\varphi_{m}\left(\theta ; k_{0}\right)\right\}_{0}^{\infty}$ is a Schauder basis of $L^{p}$ for all $k_{0} \in Q_{2}$.

Step Four: We shall now prove that the pair $\left(L^{p},\left\{\phi_{m}\left(\theta ; k_{0}\right)\right\}_{0}^{\infty}\right), k_{0} \in Q_{2}$, possesses $\ell^{q}$-bounded a.s.c.f. Consider the projection $P_{B}: L^{p} \rightarrow L_{B}^{p}$. Since both its range $R\left(P_{B}\right)=L_{B}^{p}$ and its null space $N\left(P_{B}\right)=L_{A}^{p}$ are closed, $P_{B}$ is continuous. Thus,

$$
\left\|u\left(\theta ; k_{0}\right)\right\|_{L_{B}^{p}}=\left\|P_{B} v\left(\theta ; k_{0}\right)\right\|_{L_{B}^{p}} \leq\left\|P_{B}\right\|\left\|v\left(\theta ; k_{0}\right)\right\|_{L^{p}},
$$

which, in conjunction with (4.22), gives

$$
\left(\sum_{m=2}^{\infty}\left|h_{m}^{*}\left(u ; k_{0}\right)\right|^{q}\right)^{1 / q} \leq A_{2}\left(Q_{2}\right)\left\|v\left(\theta ; k_{0}\right)\right\|_{L^{p}} .
$$

On the other hand, from the linear system (4.20) we obtain

$$
\left|a_{n}\left(k_{0}\right)\right| \leq D_{n}\left(Q_{2}\right)\left\|v\left(\theta ; k_{0}\right)\right\|_{L^{p}}, \quad n=0,1,
$$

which, in conjunction with (4.15), (4.24a), and (4.26), implies that

$$
\left(\sum_{m=0}^{1}\left|h_{m}\left(v ; k_{0}\right)\right|^{q}\right)^{1 / q} \leq A_{3}\left(Q_{2}\right)\left\|v\left(\theta ; k_{0}\right)\right\|_{L^{p}} .
$$

Finally, combining the inequalities (4.26) and (4.28), we obtain

$$
\left\|h_{m}\left(v ; k_{0}\right)\right\|_{\ell q} \leq C\left(Q_{2}\right)\left\|v\left(\theta ; k_{0}\right)\right\|_{L^{p}},
$$

which proves that $\left(L^{p},\left\{\phi_{m}\left(\theta ; k_{0}\right)\right\}_{0}^{\infty}\right)$ possesses $\ell^{q}$-bounded a.s.c.f.

(ii) The case $p \geq 2$. Consider first the case $p=2$. Then, although all the above arguments hold true, the basis property of $\left\{\phi_{m}\left(\theta ; k_{0}\right)\right\}_{0}^{\infty}$ can also be proved easier, using Hilbert-space techniques (cf. [3, Secs. 4, 5]). Since now $\{\cos (m \theta)\}_{0}^{\infty}$ is an orthogonal basis of $L^{2}$, it follows that $\left\{\phi_{m}\left(\theta ; k_{0}\right)\right\}_{0}^{\infty}$ is a Riesz basis of $L^{2}$ (cf. Lemma 4.1). The above results in general cannot be improved for $2<p \leq \infty$, since the Hausdorff-Young Theorem cannot be extended for this case (see Theorem 4.1 (ii)). This completes the proof of the Theorem. 
REMARK 4.1. In proving the invertibility of the operator $I-T_{k_{0}}$, in Theorem 4.3, use was made of the fact that $\left\|T_{k_{0}}\right\|<1$, for sufficiently small $\left|k_{0}\right|$. However, the invertibility of $I-T_{k_{0}}$ and thus all the conclusions of the Theorem remain true if there exists at least one $k_{0}^{*} \in Q$, for which the sequence $\left\{\phi_{m}\left(\theta ; k_{0}^{*}\right)\right\}_{0}^{\infty}$ is $\ell^{q}$-linearly independent (see Theorem 5.1 [13, p. 21]). This is equivalent to a uniqueness theorem for the problem $\mathbf{P}\left(\zeta ; k_{0}^{*}\right)$. When this is the case $k_{0}^{*} \in Q-S$.

REMARK 4.2. For Im $k_{0}>0$, a uniqueness theorem can be proved for the problem $\mathbf{P}\left(\zeta ; k_{0}\right)\left[21\right.$, p. 9]. Thus, we can assert that $S \cap\left\{k_{0}: \operatorname{Im} k_{0}>0\right\}=\varnothing$.

REMARK 4.3. The basis property of $\left\{\phi_{m}\left(\theta ; k_{0}\right)\right\}_{0}^{\infty}$ holds true for all $k_{0} \in \mathbb{R}^{+}-F$, where $F$ is a set of isolated points. However, the existing experience permits us to conjecture that $F=\varnothing$; see, e.g., [33].

REMARK 4.4. The property that the pair $\left(L^{p},\left\{\phi_{m}\left(\theta ; k_{0}\right)\right\}_{0}^{\infty}\right)$ possesses $\ell^{q}$-bounded a.s.c.f. is, in fact, equivalent with the boundedness of the resolvent operator of the problem $\mathbf{P}\left(\mathbf{D} ; k_{0}\right)$, which to any given forcing term $\mathbf{V}\left(\theta ; k_{0}\right)$ (see $\left.(3.14)\right)$ corresponds its coefficients $h_{m}\left(\mathbf{V} ; k_{0}\right)$, (see $\left.(4.23)\right)$, with respect to the basis $\left\{\phi_{m}\left(\theta ; k_{0}\right)\right\}_{0}^{\infty}$.

We conclude this section by stating a theorem concerning the $k_{0}$-analyticity of the associated to $\left\{\phi_{m}\left(\theta ; k_{0}\right)\right\}_{0}^{\infty}$ coefficient functionals.

TheOREM 4.4. Let $Q \subset \mathbb{C}_{j}$ be an open set for which the sequence $\left\{\phi_{m}\left(\theta ; k_{0}\right)\right\}_{0}^{\infty}$, $k_{0} \in Q$, is a basis of $L^{p}(-\pi, 0)$. Then, for any given $x(\theta) \in L^{p}(-\pi, 0)$, its coefficient functionals $h_{m}\left(x ; k_{0}\right), m=0,1, \ldots$, are analytic with respect to $k_{0} \in Q$. Moreover, if $x\left(\theta ; k_{0}\right) \in L^{p}(-\pi, 0)$ is analytic with respect to $k_{0} \in Q$, its coefficient functionals $h_{m}^{*}\left(k_{0}\right)=h_{m}\left(x\left(\theta ; k_{0}\right) ; k_{0}\right)$ are also analytic in $Q$.

The proof of this Theorem is simple and so it will be omitted (see [7, pp. 37-38]).

5. The solvability of the problem $\mathbf{P}\left(\zeta ; k_{0}\right)$. Theorem 4.3 immediately implies that, for $\partial D_{B} \in W^{1, p}, p>1$, and for a.a. $k_{0} \in \mathbb{C}_{j}-\{0\}$, the problem $\mathbf{P}\left(\mathbf{D} ; k_{0}\right)$ admits a unique solution in the sequence space $\ell^{q^{*}}, q^{*}=\max \{2, p /(p-1)\}$, and that the resolvent operator is bounded (see Remark 4.4). We now proceed to establish the solvability of the problem $\mathbf{P}\left(\zeta ; k_{0}\right)$.

TheOREM 5.1. Let $\partial D_{B} \in W^{1, p}, 1<p \leq \infty$, and let $q=p /(p-1)$ be the conjugate exponent. Let also $k_{0} \in Q-S$, where $Q, S$ are as in Theorem 4.3 (in the physical problem $\left.k_{0} \in(0, \infty)\right)$. Then, there exists a unique $j$-complex sequence $\left\{D_{m}\left(k_{0}\right)\right\}_{0}^{\infty} \in$ $\ell^{q^{*}}, q^{*}=\max \{2, q\}$, defining the $i j$-complex function

$$
\mathbf{F}\left(\zeta ; k_{0}\right)=D_{0}\left(k_{0}\right) \mathbf{G}_{0}\left(\zeta ; k_{0}\right)+D_{1}\left(k_{0}\right) \mathbf{G}_{1}\left(\zeta ; k_{0}\right)-\sum_{m=2}^{\infty}\left(D_{m}\left(k_{0}\right) / m\right) \mathbf{M}_{m}\left(\zeta ; k_{0}\right)
$$

which has the following properties

(i) It is $\zeta$-analytic in $K^{-}$and satisfies conditions (3.5a, c, d). Moreover, it is extended as a single-valued $\zeta$-analytic function over any simply connected cover of $K^{-}$, say $K_{1}$, not intersecting the closed unit disk;

(ii) It is continuous on $K^{-} \cup \partial K_{B}^{-}$; 
(iii) The nontangential limit $\lim _{\zeta \rightarrow \exp (i \theta)}\left(d \mathbf{F}\left(\zeta ; k_{0}\right) / d \zeta\right)$ exists for a.a. $\theta \in[-\pi, 0]$ and defines a function belonging to $L^{P^{*}}(-\pi, 0), p^{*}=\min \{p, 2\}$, which satisfies the body-boundary condition (3.5b) a.e. and in $L^{p^{*}}$-sense;

(iv) It is analytic with respect to $k_{0} \in Q-S$;

(v) If an $\operatorname{arc} \Gamma$ of $\partial D_{B}^{-}$not containing the end points $B_{\ell}, B_{r}$, is Dini-smooth [28, p. 298], then the function $\mathbf{F}\left(\zeta ; k_{0}\right)$ satisfies the boundary condition (3.5b) pointwise in $\gamma=f^{-1}(\Gamma)$, and uniformly in closed subarcs of $\gamma$.

Proof. According to the hypotheses, the forcing term $\mathbf{V}\left(\theta ; k_{0}\right)$ in the bodyboundary condition belongs to $L^{p}(-\pi, 0)$; see Lemma 3.1. On the other hand, according to Theorem 4.3 , the sequence $\left\{\phi_{m}\left(\theta ; k_{0}\right)\right\}_{0}^{\infty}$ is a basis in $L^{p^{*}}(-\pi, 0), p^{*}=$ $\min \{p, 2\}$, and thus there exists a unique $j$-complex sequence $\left\{D_{m}\left(k_{0}\right)\right\}_{0}^{\infty}$, such that

$$
\mathbf{V}\left(\theta ; k_{0}\right)=\sum_{m=0}^{\infty} D_{m}\left(k_{0}\right) \phi_{m}\left(\theta ; k_{0}\right), \quad \text { in } L^{p^{*}}(-\pi, 0),
$$

with

$$
\left\|D_{m}\left(k_{0}\right)\right\|_{\ell^{*}} \leq A\left\|\mathbf{V}\left(\theta ; k_{0}\right)\right\|_{L^{*}}, \quad 1 / p^{*}+1 / q^{*}=1 .
$$

Consider now the function $\mathbf{F}\left(\zeta ; k_{0}\right)$ defined by (5.1). Then

(i) $\mathbf{F}\left(\zeta ; k_{0}\right)$ is $\zeta$-analytic in $K^{-}$, and satisfies conditions $(3.5 \mathrm{a}, \mathrm{c}, \mathrm{d})$ because of the Expansion Theorem 3.1. Its extensibility on $K_{1}$ follows at once from (5.1), (3.7), and (3.9).

(ii) Clearly, the functions $\mathbf{G}_{m}\left(\zeta ; k_{0}\right), m=0,1$, are continuous in $K^{-} \cup \partial K_{B}^{-}$. Since now $f^{\prime}(\zeta) \in H^{p}(K)$, it follows that $\mathbf{M}_{m}\left(\zeta ; k_{0}\right), m=2,3, \ldots$, are also continuous and moreover uniformly bounded with respect to $m$, for $\zeta \in K^{-} \cup \partial K_{B}^{-}$. On the other hand, from (5.3) we obtain that $\left\{D_{m} / m\right\} \in \ell^{1}$ and thus the series $\sum_{m=2}^{\infty} D_{m} \mathbf{M}_{m}\left(\zeta ; k_{0}\right) / m$ is uniformly convergent and represents a continuous function on $K^{-} \cup \partial K_{B}^{-}$.

(iii) Assuming that $|\zeta|>1$ and differentiating (5.1) we obtain

$$
\zeta \mathbf{F}^{\prime}\left(\zeta ; k_{0}\right)=\mathbf{H}_{A}\left(\zeta ; k_{0}\right)+\mathbf{H}_{B}\left(\zeta ; k_{0}\right)+\mathbf{H}_{C}\left(\zeta ; k_{0}\right),
$$

where

$$
\begin{gathered}
\mathbf{H}_{A}\left(\zeta ; k_{0}\right)=\zeta f^{\prime}(\zeta) \sum_{m=0}^{1} D_{m}\left(k_{0}\right) d G_{m}\left(w ; k_{0}\right) /\left.d w\right|_{w=f(\zeta)}, \\
\mathbf{H}_{B}\left(\zeta ; k_{0}\right)=\sum_{m=2}^{\infty} D_{m}\left(k_{0}\right) \zeta^{-m}, \\
\mathbf{H}_{C}\left(\zeta ; k_{0}\right)=i k_{0} \zeta f^{\prime}(\zeta) \sum_{m=2}^{\infty}\left(D_{m}\left(k_{0}\right) / m\right) \zeta^{-m} .
\end{gathered}
$$

We observe that $\mathbf{H}_{C}\left(\zeta ; k_{0}\right) \in H^{p}(K)$ and $H_{A}\left(e^{i \theta} ; k_{0}\right) \in L^{p}\left(\partial K_{B}^{-}\right)$. Note, however, that $\mathbf{H}_{A}\left(\zeta ; k_{0}\right) \notin H^{p}(K)$, since it is not single-valued in $K$. On the other hand, since the series in (5.2) converges in $L^{p^{*}}(-\pi, 0)$, and using the M. Riesz theorem for the conjugate function [12, p. 54], we conclude that $\mathbf{H}_{B}\left(\zeta ; k_{0}\right) \in H^{p^{*}}(K)$. Then, we have that the nontangential limit of the function $\zeta \mathbf{F}^{\prime}\left(\zeta ; k_{0}\right)$, as $\zeta \rightarrow e^{i \theta} \in \partial K_{B}^{-}$, exists a.e. 
and in the $L^{p^{*}}$-sense $[12$, pp. 21,34$]$, and the body-boundary condition $(3.5 b)$ is satisfied a.e. and in the $L^{p^{*}}$-sense.

(iv) Since $\left\{\phi_{m}\left(\theta ; k_{0}\right)\right\}_{0}^{\infty}$ is an analytic family of bases of $L^{p^{*}}(-\pi, 0)$ for $k_{0} \in Q-S$, we can apply Theorem 4.4 obtaining that the coefficients $D_{m}\left(k_{0}\right), m=0,1,2, \ldots$, are analytic functions of $k_{0} \in Q-S$. This fact, in conjunction with the uniform convergence of the series in $(5.1)$, implies that $\mathbf{F}\left(\zeta ; k_{0}\right)$ is an analytic function of $k_{0} \in Q-S$.

(v) Under the imposed smoothness assumptions for the arc $\Gamma$ we have that $f^{\prime}\left(e^{i \theta}\right)$ is continuous and different from zero in $\gamma$ [44], [28, p. 298]. Thus, the forcing term $\mathbf{V}\left(\theta ; k_{0}\right)$, and the boundary functions $\mathbf{H}_{A}\left(e^{i \theta} ; k_{0}\right)$ and $\mathbf{H}_{C}\left(e^{i \theta} ; k_{0}\right)$ are continuous in $\gamma$. The function $\mathbf{H}_{B}\left(\zeta ; k_{0}\right) \in H^{p^{*}}(K)$, and thus it may be represented as the Poisson integral of its boundary values $\mathbf{H}_{B}\left(e^{i \theta} ; k_{0}\right)$, which belong to $L^{p^{*}}\left(\partial K_{B}^{-}\right)$. But for $e^{i \theta} \in \gamma$ we have that

$$
\operatorname{Re}_{i}\left\{\mathbf{H}_{B}\left(e^{i \theta} ; k_{0}\right)\right\}=\mathbf{V}\left(\theta ; k_{0}\right)-\operatorname{Re}_{i}\left\{\mathbf{H}_{A}\left(e^{i \theta} ; k_{0}\right)\right\}-\operatorname{Re}_{i}\left\{\mathbf{H}_{C}\left(e^{i \theta} ; k_{0}\right)\right\},
$$

which implies that $\operatorname{Re}_{i}\left\{\mathbf{H}_{B}\left(\zeta ; k_{0}\right)\right\}$ is continuous in $\gamma$, and, according to [37, p. 130], $\operatorname{Re}_{i}\left\{\mathbf{H}_{B}\left(\zeta ; k_{0}\right)\right\}$ is continuous in $K^{-} \cup \gamma$. This fact, in conjunction with the continuity of $\mathbf{H}_{A}\left(\zeta ; k_{0}\right)$ and $\mathbf{H}_{C}\left(\zeta ; k_{0}\right)$ in $K^{-} \cup \gamma$, completes the proof of the part (v) of the Theorem.

We shall conclude this section by commenting on the best possible character of our results and by comparing our approach with the infinite-system approach by Ursell $[40,42]$.

Taking into account (3.17) we can write (5.2) in the form

$$
\begin{aligned}
& D_{0}\left(k_{0}\right) \phi_{0}\left(\theta ; k_{0}\right)+D_{1}\left(k_{0}\right) \phi_{1}\left(\theta ; k_{0}\right) \\
& \quad+\sum_{m=2}^{\infty} D_{m}\left(k_{0}\right) \cos (m \theta)+k_{0} \sum_{m=2}^{\infty}\left(D_{m}\left(k_{0}\right) / m\right) g_{m}(\theta)=\mathbf{V}\left(\theta ; k_{0}\right) .
\end{aligned}
$$

Then, taking the cosine-Fourier coefficients of the two members of (5.6), we obtain the following infinite linear system (which also can be used for numerical calculations; see [6]):

$$
\begin{array}{r}
D_{0}\left(k_{0}\right) \tilde{\phi}_{0 c}\left(n ; k_{0}\right)+D_{1}\left(k_{0}\right) \tilde{\phi}_{1 c}\left(n ; k_{0}\right)+k_{0} \sum_{m=2}^{\infty}\left(D_{m}\left(k_{0}\right) / m\right) \tilde{g}_{m c}(n)=\tilde{\mathbf{V}}_{c}\left(n ; k_{0}\right), \\
n=0,1, \\
\begin{array}{r}
D_{0}\left(k_{0}\right) \tilde{\phi}_{0 c}\left(n ; k_{0}\right)+D_{1}\left(k_{0}\right) \tilde{\phi}_{1 c}\left(n ; k_{0}\right)+\left(D_{n}\left(k_{0}\right) / 2\right)+k_{0} \sum_{m=2}^{\infty}\left(D_{m}\left(k_{0}\right) / m\right) \tilde{g}_{m c}(n) \\
=\widetilde{\mathbf{V}}_{c}\left(n ; k_{0}\right), \quad n \geq 2,
\end{array}
\end{array}
$$

where

$$
\tilde{g}_{m c}(n)=-\left[\tilde{x}_{2 c}^{\prime}(m-n)+\tilde{x}_{2 c}^{\prime}(m+n)+\tilde{x}_{3 s}^{\prime}(m-n)+\tilde{x}_{3 s}^{\prime}(m+n)\right] / 2,
$$

and, for any integrable function $f(\theta)$, the symbol $\tilde{f}_{c}(n)$ denotes the $n$ th-order cosineFourier coefficient of $f(\theta), \theta \in[-\pi, 0]$, defined by Eq. (3.27), while the symbol $\tilde{f}_{s}(n)$ denotes the corresponding sine-Fourier coefficient defined similarly. (For example, 
$\tilde{x}_{2 c}^{\prime}(n)$ denotes the $n$ th-order cosine-Fourier coefficient of the function $\left.x_{2}^{\prime}(\theta)\right)$. Equation (5.7b) shows that $O\left(D_{n}\left(k_{0}\right)\right)$ is dominated by $O\left(\tilde{\mathbf{V}}_{c}\left(n ; k_{0}\right)\right)$, and this implies that the estimate $\left\{D_{n}\left(k_{0}\right)\right\}_{0}^{\infty} \in \ell^{q^{*}}$ is the best possible. (See the comments after the proof of Theorem 3.2.)

Moreover, one would examine the solvability question of the problem $\mathbf{P}\left(\zeta ; k_{0}\right)$ by studying the solvability of the infinite linear system $(5.7 \mathrm{a}, \mathrm{b})$. Such an approach was introduced by Ursell [40, p. 93] for the case of a floating semi-circular cylinder (see also [42, p. 293]), and extended to floating bodies with analytic boundaries by Athanassoulis [3, Sec. 6]. In the above works the solvability of the system (5.7) was proved in the Hilbert-space $\ell^{2}$ and this had been made possible since $O\left(\tilde{\mathbf{V}}_{c}(n)\right)=O\left(n^{-2}\right)$. For nonsmooth body boundaries, e.g., when $\partial D_{B} \in W^{1, p}, p>1$, the solvability of the linear system (5.7) should be justified in $\ell^{q^{*}}, q^{*}=p^{*} /\left(p^{*}-1\right)$. For this, we have to prove the compactness of the corresponding infinite-matrix operator in $\ell^{q^{*}}$, that is, we have to prove that

$$
\sum_{n=2}^{\infty}\left(\sum_{m=2}^{\infty}\left|\tilde{g}_{m c}(n) / m\right|^{p^{*}}\right)^{q^{*} / p^{*}}<\infty
$$

The proof of the convergence of the above double series, without having at our disposal individual estimates for $\tilde{g}_{m c}(n)$, is a very difficult task, which the present authors have not yet succeeded in carrying out.

The above observations permit us to compare our basis-theory approach to the more traditional infinite-system approach. When using the former approach, the main step is to prove the compactness of the operator $T_{k_{0}}$, defined in (4.17), and this can be easily carried out by examining the convergence of a simple single series (cf. Step Two of the proof of Theorem 4.3). When using the infinite-system approach, we have to prove the convergence of the double infinite series (5.9), which is an open question for body boundaries of the class $W^{1, p}$.

6. Regularity results. In this section, restricting ourselves to the radiation problem for more useful and conventional body boundaries, we shall obtain individual estimates for the sequence $\left\{D_{m}\right\}_{0}^{\infty}{ }^{3}$ improving the crude general estimate $\left\{D_{m}\right\}_{0}^{\infty} \in \ell^{q^{*}}$ obtained in Sec. 4. Consider first the case of a polygonal boundary $\partial D_{B}$.

TheOREM 6.1. Let $\partial D_{B}=\partial D_{B}^{-} \cup \partial D_{B}^{+}$be a polygonal boundary the smaller exterior angle of which is $\alpha \pi, \alpha \neq 1,0<\alpha<2$. Then, $D_{n}=O\left(n^{-\alpha}\right)$, and this is the best possible estimate.

Proof. Consider first the case where $0<\alpha<1$ (the critical case). Then $w^{\prime}(\theta) \in$ $L^{p}(-\pi, \pi)$ for $p \in[1,1 /(1-\alpha)]$, and, according to the Hausdorff-Young Theorem, $\left\{\tilde{x}_{k^{*}}^{\prime}(n)\right\}_{0}^{\infty} \in \ell^{q}, k=2,3,4,{ }^{4}$ where $q \in\left(q^{*}, \infty\right)$ and $q^{*}=\max \{2,1 / \alpha\}$ (cf. Theorem

\footnotetext{
${ }^{3}$ In this section we omit the argument $k_{0}$ from all frequency-dependent quantities.

${ }^{4}$ The subscript * stands for $c$ or $s$.
} 
2.1). Taking into account (5.8) we write

$$
\begin{aligned}
D_{n} / 2= & \tilde{\mathbf{V}}_{c}(n)-D_{0} \tilde{\phi}_{0 c}(n)-D_{1} \tilde{\phi}_{1 c}(n) \\
& -k_{0} \sum_{m=2}^{\infty} D_{m}\left[\tilde{x}_{2 c}^{\prime}(m-n)+\tilde{x}_{2 c}^{\prime}(m+n)-\tilde{x}_{3 s}^{\prime}(m-n)+\tilde{x}_{3 s}^{\prime}(m+n)\right] / 2 m, \\
& n \geq 2 .
\end{aligned}
$$

To obtain individual estimates for the coefficients $D_{n}$ we shall estimate all the terms appearing in the right-hand side of $(6.1)$.

Estimation of the terms $\tilde{\mathbf{V}}_{c}(n), \tilde{\phi}_{0 c}(n), \tilde{\phi}_{1 c}(n)$. Using the explicit form of the conformal mapping function $f(\zeta)$ (obtained via the Schwarz-Christoffel transformation), we can express the Fourier coefficients of $w^{\prime}(\theta)$ in terms of the incomplete Gamma Function. Then, with the aid of some standard asymptotic expansions, we find

$$
\tilde{\mathbf{V}}_{c}(n)=O\left(n^{-\alpha}\right)
$$

and this estimate is the best possible when $0<\alpha<1$. See Appendix A.

To obtain individual estimates for $\tilde{\phi}_{k c}(n), k=0,1$, we observe that we actually have to estimate the cosine-Fourier coefficients of functions of the type $h(\theta)=$ $w^{\prime}(\theta) F(w(\theta))$, (see $\left.(3.16 \mathrm{a}, \mathrm{b}, \mathrm{c})\right)$ where $F(w(\theta))$ is infinitely differentiable with respect to $w(\theta)$, and absolutely continuous with respect to $\theta$. Thus, we have

$$
\widetilde{F}_{c}(n)=o\left(n^{-1}\right), \quad \widetilde{F}_{c}^{1}(n)=o\left(n^{-1}\right) .
$$

The estimate (6.3a) can be improved as follows. Using an integration by parts we obtain

$$
\widetilde{F}_{c}(n)=-\frac{1}{n \pi} \int_{-\pi}^{0} \frac{d F(w(\theta))}{d w} w^{\prime}(\theta) \sin (n \theta) d \theta .
$$

Now applying the Theorem $\mathrm{Cl}$ (Appendix $\mathrm{C}$ ) concerning the Fourier coefficients of the product of two functions, and taking into account the estimates (6.3b) and (A10) (Appendix A), we find that

$$
\widetilde{F}_{c}(n)=O\left(n^{-(1+\alpha-\varepsilon)}\right),
$$

with arbitrarily small $\varepsilon>0$. After establishing (6.5) we can apply once more Theorem $\mathrm{C} 1$ to obtain

$$
\tilde{h}_{c}(n)=O\left(n^{-\alpha}\right)
$$

Now, using (6.6) in conjunction with $(3.16 \mathrm{a}, \mathrm{b}, \mathrm{c})$ we easily find

$$
\tilde{\phi}_{0 c}(n)=O\left(n^{-\alpha}\right), \quad \tilde{\phi}_{1 c}(n)=O\left(n^{-\alpha}\right) .
$$

Estimation of the terms $\sum D_{m} \tilde{x}_{k *}^{\prime}(m \pm n) / m, k=2,3$. Using the Hölder inequality, and the estimates $\left\{D_{m}\right\} \in l^{q}, q^{*}<q<\infty$, and (A10) we obtain

$$
\begin{aligned}
\sum_{m=2}^{\infty}\left|D_{m} \tilde{x}_{k *}^{\prime}(m \pm n) / m\right| & \leq\left(\sum_{m=2}^{\infty}\left|D_{m}\right|^{q}\right)^{1 / q}\left(\sum_{m=2}^{\infty}\left|\tilde{x}_{k *}^{\prime}(m \pm n) / m\right|^{p}\right)^{1 / p} \\
& \leq A\left(\sum_{m=2}^{\infty}{ }^{\prime} m^{-p}|m \pm n|^{\alpha p}\right)^{1 / p}
\end{aligned}
$$


where the prime in the summation symbol indicates that the term $|m-n|^{-\alpha p}$ corresponding to $m=n$ is omitted from the summation. The estimation of the last series is difficult and laborious and is given without proof in Appendix B. The result is

$$
\sum_{m=2}^{\infty} D_{m} \tilde{x}_{k *}^{\prime}(m \pm n) / m=O\left(n^{-\alpha}\right) .
$$

Now combining (6.2), (6.7), and (6.9) we obtain the estimate

$$
D_{n}=O\left(n^{-\alpha}\right), \quad 0<\alpha<1 .
$$

It should be emphasized that, since the estimate (6.2) is strict, the individual estimate (6.10) for the coefficients $D_{n}$ is, in general, the best possible. Let it also be noted that Eq. (6.1) cannot give better estimates than $D_{n}=O\left(n^{-1}\right)$, however smooth the boundary $\partial D_{B}$ is.

Now consider the case $1<\alpha<2$ (the noncritical case). In this case $\left|w^{\prime}(\theta)\right| \in$ $C[-\pi, \pi]$, while $w^{\prime \prime}(\theta) \in L^{p}(-\pi, \pi)$, for $p \in[1,1 /(2-\alpha))$. Thus, by performing an integration by parts in (6.1), we obtain

$$
\begin{aligned}
D_{n} / 2= & \tilde{\mathbf{V}}_{c}(n)-D_{0} \tilde{\phi}_{0 c}(n)-D_{1} \tilde{\phi}_{1 c}(n) \\
& +\left(k_{0} / 2 n\right) \sum_{m=2}^{\infty}\left(D_{m} / m\right)\left(\tilde{x}_{2 s}^{\prime \prime}(n-m)+\tilde{x}_{2 s}^{\prime \prime}(n+m)+\tilde{x}_{3 c}^{\prime \prime}(n-m)+\tilde{x}_{3 c}^{\prime \prime}(n+m)\right) \\
& +\left(k_{0} / 2 n\right) \sum_{m=2}^{\infty} D_{m}\left(-\tilde{x}_{2 c}^{\prime}(n-m)+\tilde{x}_{2 c}^{\prime}(n+m)+\tilde{x}_{3 s}^{\prime}(n-m)+\tilde{x}_{3 s}^{\prime}(n+m)\right) .
\end{aligned}
$$

We now proceed to estimate the terms appearing in the right-hand side of (6.11). For the first three we can use the same arguments as for the critical case. Thus, taking into account the estimates (A15), (A16a), and (A19) we obtain

$$
\tilde{\mathbf{V}}_{c}(n)=O\left(n^{-\alpha}\right), \quad \tilde{\phi}_{0 c}(n)=O\left(n^{-\alpha}\right), \quad \tilde{\phi}_{1 c}(n)=O\left(n^{-\alpha}\right), \quad(6.12 \mathrm{a}, \mathrm{b}, \mathrm{c})
$$

where now $1<\alpha<2$. The estimation of the first four infinite series containing $\tilde{x}_{k *}^{\prime \prime}(n \pm m), k=2,3$, is accomplished by using the Cauchy-Schwartz inequality since now $\left\{D_{m}\right\}_{0}^{\infty} \in \ell^{2}$. Then, taking into account (A15), (A16a), and the results of Appendix B, we find

$$
(1 / n) \sum_{m=2}^{\infty} D_{m} \tilde{x}_{k *}^{\prime \prime}(n \pm m) / m=O\left(n^{-\alpha}\right), \quad k=2,3 .
$$

Estimation of the terms $\sum D_{m} \tilde{x}_{k *}^{\prime}(n \pm m), k=2,3$. Estimating the terms of Eq. (6.1) as in the critical case, we easily obtain that $D_{m}=O\left(m^{-1}\right)$. If we set $\Psi_{m}=$ $m^{\alpha-1} D_{m}, m \geq 2$, then, $\Psi_{m}=O\left(m^{\alpha-2}\right)$ and $\left\{\Psi_{m}\right\} \in \ell^{q}$ with $q>1 /(2-\alpha)$. Now using the Hölder inequality and taking into account the estimates (A15) and (A16b) 
we obtain

$$
\begin{aligned}
\sum_{m=2}^{\infty}\left|D_{m} \tilde{x}_{k *}^{\prime}(n \pm m)\right| & =\sum_{m=2}^{\infty}\left|\Psi_{m} \tilde{x}_{k *}^{\prime}(n \pm m) / m^{\alpha-1}\right| \\
& \leq\left(\sum_{m=2}^{\infty}\left|\Psi_{m}\right|^{q}\right)^{1 / q}\left(\sum_{m=2}^{\infty}\left|\tilde{x}_{k *}^{\prime}(n \pm m) / m^{\alpha-1}\right|^{p}\right)^{1 / p} \\
& \leq A\left(\sum_{m=2}^{\infty}{ }^{\prime} m^{-(\alpha-1) p}|n \pm m|^{-p}\right)^{1 / p}
\end{aligned}
$$

which, in conjunction with the results of Appendix B, gives

$$
(1 / n) \sum_{m=2}^{\infty} D_{m} \tilde{x}_{k *}^{\prime}(n \pm m)=O\left(n^{-\alpha}\right), \quad k=2,3 .
$$

Combining now (6.12), (6.13), and (6.14) we obtain the estimate

$$
D_{n}=O\left(n^{-\alpha}\right), \quad 1<\alpha<2,
$$

which is the best possible, since estimate $(6.12 \mathrm{a})$ is strict. This completes the proof of the theorem.

REMARK 6.1. Exploiting some classical results about the boundary behaviour of the conformal mapping function in the vicinity of the corner points [23], we can prove that the estimate of Theorem 6.1 remains true for piecewise smooth boundaries $\partial D_{B}$, consisting of sufficiently smooth arcs (curvilinear polygons).

REMARK 6.2. A simple consequence of the estimate $D_{n}=O\left(n^{-\alpha}\right)$ is that the transformed velocity field is continuous on $\partial K_{B}^{-}$in the noncritical case (i.e., $1<$ $\alpha<2$ ), while it might become unbounded at the points of $\partial K_{B}^{-}$corresponding to the corner points in the critical case (i.e., $0<\alpha<1)$. These conclusions are reversed in the physical plane, as can be seen by means of the relation $F^{\prime}\left(w ; k_{0}\right)=\mathbf{F}^{\prime}\left(\zeta ; k_{0}\right) / f^{\prime}(\zeta)$.

When the boundary $\partial D_{B}$ is sufficiently smooth the estimate for the coefficients $D_{n}$ becomes $D_{n}=O\left(n^{-2}\right)$. In this connection we introduce the following

Definition 6.1. We shall say that the boundary $\partial D_{B}$ belongs to $\mathbf{C}^{2, *}$ if $f^{\prime \prime}\left(e^{i \theta}\right)$ is continuous and of bounded variation for $\theta \in[-\pi, \pi]$.

For boundaries $\partial D_{B} \in \mathbf{C}^{2, *}$ we have the following

Theorem 6.2. Let $\partial D_{B} \in \mathrm{C}^{2, *}$. Then, $D_{n}=O\left(n^{-2}\right)$ and this estimate is the best possible, i.e., it cannot be improved however smooth the boundary is (cf. [42] and [3]).

Proof. The estimation of $D_{n}$ is again based on the relations (6.1), which leads directly to $D_{n}=O\left(n^{-1}\right)$, and (6.11), which, by means of a procedure very similar to that used in the case of noncritical angles in Theorem 6.1, successively gives first $D_{n}=O\left(n^{-2+\varepsilon}\right)$ and finally $D_{n}=O\left(n^{-2}\right)$. In accomplishing the proof use is made of the estimates (A20)-(A23). The best possible character of the estimate $D_{n}=O\left(n^{-2}\right)$ is a direct consequence of the fact that the estimates (A20), (A21a), (A22b), and (A23a) cannot be improved; see (A24). 
Appendix A. Estimation of the Fourier coefficients of $x_{k}^{\prime}(\theta), k=2,3,4$. Consider the conformal mapping function $f(\zeta)$ transforming the exterior unit disk onto the exterior of a polygonal section $\partial D_{B}[27]$

$$
f(\zeta)=P \int_{\zeta_{0}}^{\zeta} \prod_{k=-n+1}^{n}\left(\zeta-\zeta_{k}\right)^{\alpha_{k}-1} \frac{d \zeta}{\zeta^{2}}+C .
$$

$P, C$ are properly determined constants, and $\zeta_{k}=\exp \left(i \beta_{k}\right), k=-n+1,-n+2, \ldots, n$, are the points on the unit circle $\partial K_{B}$, corresponding to the vertices $A_{k}$ of the polygon $\partial D_{B}$ (see Fig. 2).

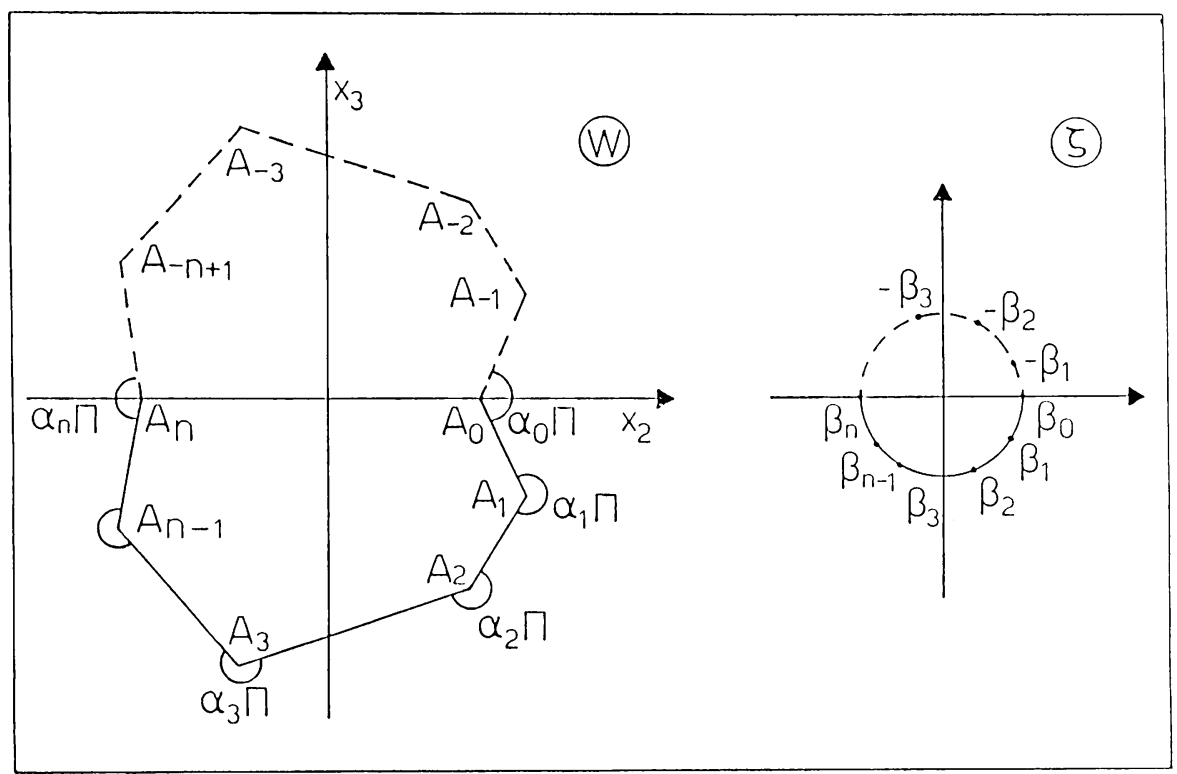

FIG. 2

In accordance with the symmetry of $\partial D_{B}$ with respect to the $x_{2}$-axis, we have $\beta_{0}=0, \beta_{n}=-\pi, \beta_{-k}=-\beta_{k}, k=1, \ldots, n-1$. Now, taking into account (2.6) we obtain

$$
w^{\prime}(\theta)=x_{2}^{\prime}(\theta)+i x_{3}^{\prime}(\theta)=i P \prod_{k=-n+1}^{n}\left(\exp (i \theta)-\exp \left(i \beta_{k}\right)\right)^{\alpha_{k}-1} \exp (-i \theta),
$$

from which

$$
\left|w^{\prime}(\theta)\right|=|P| \prod_{k=-n+1}^{n}\left|1-\exp \left(i\left(\beta_{k}-\theta\right)\right)\right|^{\alpha_{k}-1}=4|P| \prod_{k=-n+1}^{n}\left|\sin \left(\left(\beta_{k}-\theta\right) / 2\right)\right|^{\alpha_{k}-1} .
$$

Note that $w^{\prime}(\theta)$ is continuous at all points $\zeta$ of the unit circle $\partial K_{B}$, except from the points $\zeta_{k}=\exp \left(i \beta_{k}\right)$ corresponding to vertices of acute exterior angles, i.e., angles with $\alpha_{k}<1$ (critical angles). 
We shall now estimate the Fourier coefficients of $x_{k}^{\prime}(\theta), k=2,3,4$. For this, we have to estimate, with respect to $m$, the integrals

$$
\int_{-\pi}^{0} w^{\prime}(\theta)\left\{\begin{array}{l}
\sin (m \theta) \\
\cos (m \theta)
\end{array} d \theta=\int_{-\pi}^{-\pi+\varepsilon} \bullet+\int_{-\varepsilon}^{0} \bullet+\sum_{k=1}^{n-1} \int_{\beta_{k}-\varepsilon}^{\beta_{k}+\varepsilon} \bullet+\sum_{k=1}^{n} \int_{b_{k}+\varepsilon}^{\beta_{k-1}-\varepsilon} \bullet\right.
$$

Now taking into account (A3) and the fact that $\operatorname{Arg}\left\{w^{\prime}(\theta)\right\}$ is piecewise constant, we conclude that the estimation of the integrals appearing in the right-hand side of (A4) is reduced to the estimation of integrals of the form

$$
\int_{a}^{b}\left|w^{\prime}(\theta)\right| \phi_{m}(\theta) d \theta
$$

where $\phi_{m}(\theta)=\sin (m \theta)$ or $\cos (m \theta), m=0,1,2, \ldots$

In the neighborhood $\left(\beta_{\ell}-\varepsilon, \beta_{\ell}+\varepsilon\right), \ell=1, \ldots, n-1$, we have

$$
\begin{aligned}
\left|w^{\prime}(\theta)\right| & =4|P|\left|\sin \left(\left(\beta_{\ell}-\theta\right) / 2\right)\right|^{\alpha_{\ell}-1} \prod_{\substack{k=-n+1 \\
k \neq \ell}}^{n}\left|\sin \left(\left(\beta_{k}-\theta\right) / 2\right)\right|^{\alpha_{k}-1} \\
& =\left|\sin \left(\left(\beta_{\ell}-\theta\right) / 2\right)\right|^{\alpha_{\ell}-1} \cdot Q(\theta),
\end{aligned}
$$

where $Q(\theta)$ is analytic with respect to $\theta$ at $\theta=\beta_{\ell}$. Thus, we have

$$
\left|w^{\prime}(\theta)\right|=A\left|\theta-\beta_{\ell}\right|^{\alpha_{l}-1}+B(\theta)\left|\theta-\beta_{\ell}\right|^{\alpha_{\ell}}, \quad \theta \in\left(\beta_{\ell}-\varepsilon, \beta_{\ell}+\varepsilon\right),
$$

where $B(\theta)$ is analytic in the neighborhood $\left(\beta_{\ell}-\varepsilon, \beta_{\ell}+\varepsilon\right)$. Therefore, the estimation of the integrals in (A4) is reduced to the estimation of the following integrals

$$
\int_{0}^{\varepsilon}|\theta|^{\alpha_{\ell}-1} \phi_{m}(\theta) d \theta, \quad \int_{0}^{\varepsilon} B(\theta)|\theta|^{\alpha_{\ell}} \phi_{m}(\theta) d \theta, \quad \int_{\beta_{k}+\varepsilon}^{\beta_{k-1}-\varepsilon}\left|w^{\prime}(\theta)\right| \phi_{m}(\theta) d \theta .
$$

The first (and most difficult) integral can be explicitly calculated in terms of the incomplete Gamma function [16, p. 183]. Then, using an asymptotic relation for this special function $[1$, p. 263] we finally obtain the (strict) estimate

$$
\int_{0}^{\varepsilon}|\theta|^{\alpha_{\ell}-1} \phi_{m}(\theta) d \theta=O\left(m^{-\min \left\{1, \alpha_{\ell}\right\}}\right), \quad m \rightarrow \infty .
$$

The order of magnitude of the second and third integral in (A8) is $O\left(m^{-1}\right)$. This is easily obtained by an integration by parts and taking note that $B(\theta)|\theta|^{\alpha /}$ has its first derivative in $L^{p}(0, \varepsilon), p>1$, and that $\left|w^{\prime}(\theta)\right|$ is of bounded variation in $\left(\beta_{k}+\right.$ $\left.\varepsilon, \beta_{k-1}-\varepsilon\right), k=1, \ldots, n$.

Consequently, for a polygonal section of which the smaller exterior angle is $\alpha \pi$, $\alpha \neq 1,0<\alpha<2$, we have the following (strict) estimates for the Fourier coefficients of the functions $x_{k}^{\prime}(\theta)$

$$
\tilde{x}_{k *}^{\prime}(m)=O\left(m^{-\min \{r, 1\}}\right), \quad m \rightarrow \infty, k=2,3,
$$

where the subscript $*$ stands for $c$ or $s$.

In virtue of the above results and by using an integration by parts we obtain

$$
\begin{gathered}
\tilde{x}_{3 *}(m)=O\left(m^{-\min \{r+1,2\}}\right), \quad m \rightarrow \infty, \\
\tilde{x}_{2 c}(m)=O\left(m^{-\min \{a+1.2\}}\right), \quad \tilde{x}_{2 s}(m)=O\left(m^{-1}\right), \quad m \rightarrow \infty .
\end{gathered}
$$


Now using the estimates (A10), (A11), (A12), and the results of Appendix C we find

$$
\tilde{x}_{4 *}^{\prime}(m)=O\left(m^{-\min \{\alpha, 1\}}\right), \quad m \rightarrow \infty .
$$

The above estimates can be improved in the case of a polygonal boundary without critical angles (i.e., when $1<\alpha<2$ ). In this case $w^{\prime}(\theta) \in C(-\pi, 0)$ and $w^{\prime \prime}(\theta) \in$ $L^{p}(-\pi, 0)$, for $p \in[1,1 /(2-\alpha)]$. By repeating the above reasoning we can obtain the estimates

$$
\tilde{x}_{k}^{\prime \prime}(m)=O\left(m^{1-\alpha}\right), \quad m \rightarrow \infty, k=2,3,1<\alpha<2,
$$

from which, by an integration by parts we find

$$
\begin{array}{lll}
\tilde{x}_{2 *}^{\prime}(m)=O\left(m^{-\alpha}\right), & & m \rightarrow \infty, 1<\alpha<2, \\
\tilde{x}_{3 c}^{\prime}(m)=O\left(m^{-\alpha}\right), & \tilde{x}_{3 s}^{\prime}(m)=O\left(m^{-1}\right), & m \rightarrow \infty, 1<\alpha<2 .
\end{array}
$$

Now using these estimates and an integration by parts we find

$$
\begin{gathered}
\tilde{x}_{3 *}(m)=O\left(m^{-(1+\alpha)}\right), \quad m \rightarrow \infty, 1<\alpha<2, \\
\tilde{x}_{2 c}(m)=O\left(m^{-(1+\alpha)}\right), \quad \tilde{x}_{2 s}(m)=O\left(m^{-1}\right), \quad m \rightarrow \infty, 1<\alpha<2,
\end{gathered}
$$

which, in conjunction with (A15), (A16) and the results of Appendix C, give

$$
\tilde{x}_{4 *}^{\prime}(m)=O\left(m^{-\alpha}\right), \quad m \rightarrow \infty, 1<\alpha<2 .
$$

Note that since the estimates (A9) and (A14) are strict, the estimates (A10) and (A13) are the best possible in the case of polygonal boundaries with critical angles $(0<\alpha<1)$, while the estimates (A15), (A16), and (A19) are the best possible in the case of polygonal boundaries without critical angles $(1<\alpha<2)$.

In the case where the boundary $\partial D_{B}$ is sufficiently smooth, i.e., when $\partial D_{B} \in \mathbf{C}^{2, *}$, (see Def. 6.1), $w^{\prime \prime}(\theta)$ is continuous and of bounded variation for $\theta \in[-\pi, \pi]$, and thus we have that

$$
\tilde{x}_{k *}^{\prime \prime}(m)=O\left(m^{-1}\right), \quad m \rightarrow \infty, k=2,3,
$$

which, by an integration by parts, leads to

$$
\begin{aligned}
& \tilde{x}_{2 c}^{\prime}(m)=O\left(m^{-2}\right), \quad \tilde{x}_{2 s}^{\prime}(m)=O\left(m^{-2}\right), \quad m \rightarrow \infty, \quad(\mathrm{A} 21 \mathrm{a}, \mathrm{b}) \\
& \tilde{x}_{3 c}^{\prime}(m)=O\left(m^{-2}\right), \quad \tilde{x}_{3 s}^{\prime}(m)=O\left(m^{-1}\right), \quad m \rightarrow \infty . \quad(\mathrm{A} 22 \mathrm{a}, \mathrm{b})
\end{aligned}
$$

Now using these estimates in conjunction with the results of Appendix $C$ we easily find

$$
\tilde{x}_{4 c}^{\prime}(m)=O\left(m^{-2}\right), \quad \tilde{x}_{4 s}^{\prime}(m)=O\left(m^{-2}\right), \quad m \rightarrow \infty .
$$

The estimates (A21a), (A22b), and (A23a) cannot be further improved, however smooth the boundary $\partial D_{B}$ is, as can be seen by integrating once again by parts. For example, for the cosine-Fourier coefficient we have

$$
\tilde{x}_{k c}^{\prime}(m)=\frac{x_{k}^{\prime \prime}(0)-(-1)^{m} x_{k}^{\prime \prime}(-\pi)}{\pi m^{2}}-\frac{\tilde{x}_{k c}^{\prime \prime \prime}(m)}{m^{2}}, \quad k=2,4 .
$$


Appendix B. Asymptotic estimation of an infinite series. We give here asymptotic estimates, for large values of $n$, of the series

$$
Q(n ; p, \alpha)=\sum_{m=1}^{\infty}{ }^{\prime} m^{-\alpha}|m-n|^{-p},
$$

where the prime indicates that the term corresponding to $m=n$ is omitted from the summation. The proof of the results given in Table B1 is difficult and laborious and will be reported elsewhere. Here we note that the series $Q(n ; p, \alpha)$ is convergent provided that $p+\alpha>1$, and that the asymptotic estimates are strict.

TABLE B1. Order of magnitude of $Q(n ; p, \alpha), n \rightarrow \infty, p+\alpha>1$.

\begin{tabular}{|c|c|c|c|}
\hline & $p<1$ & $p=1$ & $p>1$ \\
\hline$a<1$ & $n^{-(p+\alpha-1)}$ & $n^{-\alpha} \ln n$ & $n^{-\alpha}$ \\
\hline$\alpha=1$ & $n^{-p} \ln n$ & $n^{-1} \ln n$ & $n^{-1}$ \\
\hline$a>1$ & $n^{-p}$ & $n^{-1}$ & $n^{-\min \{p, \alpha\}}$ \\
\hline
\end{tabular}

Appendix C. Estimation of the Fourier coefficients of functions of the form $h(\theta)=$ $f(\theta) g(\theta)$. Let

$$
f(\theta) \sim \sum_{n=-\infty}^{+\infty} c_{n} \exp (\text { in } \theta)=\hat{f}_{c}(0) / 2+\sum_{n=1}^{\infty}\left(\hat{f}_{c}(n) \cos (n \theta)+\hat{f}_{s}(n) \sin (n \theta)\right)
$$

where $\hat{f}_{c, s}(n)$ (resp. $c_{n}$ ), are the real (resp. complex) Fourier coefficients of $f(\theta)$, $\theta \in[-\pi, \pi]$. (Cf. (3.27) where the function is restricted in $[-\pi, 0]$.) Also let $\hat{g}_{c, s}(n)$ (resp. $d_{n}$ ) and $\hat{h}_{c, s}(n)$ (resp. $\gamma_{n}$ ) be the real (resp. complex) Fourier coefficients of the functions $g(\theta)$ and $h(\theta), \theta \in[-\pi, \pi]$, respectively.

Suppose that $h(\theta)=f(\theta) g(\theta) \in L^{1}(-\pi, \pi)$. Then, we have [8, p. 76]

$$
\gamma_{n}=\sum_{k=-\infty}^{+\infty} c_{k} d_{n-k}=c_{0} d_{n}+\sum_{k=1}^{\infty} c_{k} d_{n-k}+\sum_{k=1}^{\infty} c_{-k} d_{n+k}
$$

If we assume that $f(\theta)$ and $g(\theta)$ are even functions, then $h(\theta)$ is even too, and $\hat{f}_{s}(n)=\hat{g}_{s}(n)=\hat{h}_{s}(n)=0, n>0$. But for an even function $f(\theta), \theta \in[-\pi, \pi]$, we have $\hat{f}_{c}(n)=2 \tilde{f}_{c}(n)$, and consequently the cosine-Fourier coefficients of the function $h(\theta), \theta \in[-\pi, 0]$, are given by

$$
\tilde{h}_{c}(n)=\tilde{f}_{c}(0) \tilde{g}_{c}(n)+\sum_{k=1}^{\infty} \tilde{f}_{c}(n)\left(\tilde{g}_{c}(|n-k|)+\tilde{g}_{c}(n+k)\right), \quad n \geq 0 .
$$

On the other hand, if we assume that $f(\theta)$ is even and $g(\theta)$ is odd, then $h(\theta)$ is odd too, and $\hat{f}_{s}(n)=\hat{g}_{c}(n)=\hat{h}_{c}(n)=0, n \geq 0$. Now using the relations $\hat{f}_{c}(n)=2 \tilde{f}_{c}(n)$ and $\hat{g}_{s}(n)=2 \tilde{g}_{s}(n)$, we obtain the following formula for the sine Fourier coefficients 
of the function $h(\theta), \theta \in[-\pi, 0]$,

$$
\begin{aligned}
\tilde{h}_{s}(n)= & \tilde{f}_{c}(0) \tilde{g}_{s}(n)+\sum_{k=1}^{n} \tilde{f}_{c}(k) \tilde{g}_{s}(n-k)-\sum_{k=n+1}^{\infty} \tilde{f}_{c}(k) \tilde{g}_{s}(k-n) \\
& +\sum_{k=1}^{\infty} \tilde{f}_{c}(k) \tilde{g}_{s}(n+k), \quad n>0 .
\end{aligned}
$$

Taking into account relations (C3) and (C4) and the results of Appendix B we can easily prove the following

Theorem C1. Let $h(\theta)=f(\theta) g(\theta)$ and $\tilde{f}_{c}(n)=O\left(n^{-(1+\alpha)}\right)$ and $\tilde{g}_{c, s}(n)=O\left(n^{-\beta}\right)$. Then

(i) If $\beta<1, \alpha=0$, we have $\tilde{h}_{c, s}(n)=O\left(n^{-(\beta-\varepsilon)}\right)$, with arbitrarily small $\varepsilon>0$;

(ii) If $\beta<1, \alpha>0$, we have $\tilde{h}_{c, s}(n)=O\left(n^{-\beta}\right)$;

(iii) If $\beta>1$, we have $\tilde{h}_{c, s}(n)=O\left(n^{-\min (1+\alpha, \beta)}\right)$.

The estimates (ii) and (iii) are strict.

Acknowledgment. The authors wish to thank the referee of a first version of this paper whose comments motivated the regularity results presented in Sec. 6.

\section{REFERENCES}

[1] M. Abramowitz and I. A. Stegun, Handbook of Mathematical Functions, Dover, 1972.

[2] G. A. Athanassoulis, An expansion theorem for water-wave potentials, J. Engrg. Math. 18, 181-194 (1984)

[3] G. A. Athanassoulis, On the solvability of a two-dimensional water-wave radiation problem, Quart. Appl. Math. 44, 601-620 (1987)

[4] G. A. Athanassuolis, P. D. Kaklis, and C. G. Politis, The limiting values of added masses of a partially submerged cylinder of arbitrary shape, J. Ship Research 32 1, 1-18 (1987)

[5] G. A. Athanassoulis, P. D. Kaklis, and C. G. Politis, Low-frequency oscillations of a partially submerged cylinder of arbitrary shape, National Technical University of Athens, Dept. of Naval Arch. and Marine Engrg., Report No. D1-88 (1988)

[6] G. A. Athanssoulis and C. G. Politis, On a radiation problem for two-dimensional floating bodies of arbitrary shape with corners, Scientific and Methodological Seminar on Ship Hydrodynamics, Varna, Bulgaria (1985)

[7] G. A. Athanassoulis and C. G. Politis, On the solvability of a two-dimensional wave-body interaction problem, National Technical University of Athens, Dept. of Naval Arch. and Marine Engrg., Report No. 2 (1987)

[8] N. K. Barry, A Treatise on Trigonometric Series, vol. I, Pergamon Press, 1964

[9] J. T. Beale, Eigenfunctions expansions for objects floating in an open sea, Comm. Pure Appl. Math. 30, 283-313 (1977)

[10] M. B. Count, On the hydrodynamic behaviour of ocean wave energy absorbing structures-A theoretical treatment, Central Electricity Generating Board, England, Report R/M/N960 (1977)

[11] K. Doppel and G. C. Hsiao, On weak solutions of the floating body problem, University of Delaware, Dept. of Math. Sciences, Center for the Math. of Waves, Report No. 87-17 (1987)

[12] P. L. Duren, Theory of $H^{p}$-spaces, Academic Press, 1970

[13] I. C. Gohberg and M. G. Krein, Introduction to the Theory of Linear Nonselfadjoint Operators, Trans. Math. Monographs, vol. 18, Amer. Math. Soc., 1969

[14] G. M. Goluzin, Geometric Theory of Functions of a Complex Variable, Transl. Math. Monographs, vol. 26, Amer. Math. Soc., 1969

[15] P. Guevel and J. M. Kobus, Flotteurs cylindriques horizontaux soumis à des oscillations forcées de très faibles amplitudes, Bulletin d' Association Technique Maritime et Aeronautique 75, 183-204 (1975)

[16] I. S. Gradshteyn and I. M. Ryzhik, Table of Integrals, Series, and Products, Academic Press, 1965 
[17] D. F. Harazov, On the spectrum of completely continuous operators depending analytically on a parameter, in topological linear spaces, (Russian), Acta Sci. Math. (Szeged) 23, 38-45 (1962)

[18] F. John, On the motion of floating bodies. II: Simple harmonic motions, Comm. Pure Appl. Math. 3, 45-100 (1950)

[19] P. D. Kaklis and G. A. Athanassoulis, The low-frequency heaving motion of a cylinder on the free surface of a liquid, SIAM J. Appl. Math. 49 2, 1-17 (1987)

[20] Y. Katznelson, An Introduction to Harmonic Analysis, Dover, 1968

[21] R. E. Kleinman, On the mathematical theory of the motion of floating bodies-an update, David Taylor Naval Ship Research and Development Center, Report 82/074 (1982)

[22] N. G. Kuznetsov and V. G. Maz'ya, Problem concerning steady state oscillations of a layer of fluid in the presence of an obstacle, Soviet Phys. Dokl. 19, 341-343 (1974)

[23] M. Lavrentiev and B. Chabat, Effet hydrodynamique et modèles mathémtiques, Editions MIR, Moscou, 1980

[24] M. Lenoir, Une application du principe d'absorption limite au problem bidimensionnel du mouvement sur la houle, in Méthodes de couplage en hydrodynamique navale et application à la résistance de vagues bidimensionnele, École Nationale Supérieure de Techniques Avancées, Rapport de Recherche 164 (1982), pp. III.1-III.29

[25] M. Lenoir and D. Martin, An application of the principle of limiting absorption to the motion of floating bodies, J. Math. Anal. Appl. 79, 370-383 (1981)

[26] F. D. Lesley, Personal communication, 1985

[27] A. I. Markushevich, Theory of Functions of a Complex Variable, Chelsea Publ. Co., 1977

[28] C. Pommerenke, Univalent Functions, Vandenhoeck \& Ruprecht, Göttingen, 1975

[29] C. Pommerenke, On univalent functions, Bloch functions and VMOA, Math. Ann. 236, 199-208 (1978)

[30] C. Pommerenke, Personal communication, 1985

[31] F. Reisz and B. Sz. -Nagy, Functional Analysis, Frederick Ungar Publ. Co., 1955

[32] G. E. Schober, Personal communication, 1985

[33] M. J. Simon and F. Ursell, Uniqueness in linearized two-dimensional water-wave problems, J. Fluid Mech. 148, 137-154 (1984)

[34] I. Singer, Bases in Banach spaces I, Springer-Verlag, 1970

[35] S. Steinberg, Meromorphic families of compact operators, Arch. Rational Mech. Anal. 31, 372-380 (1968)

[36] A. E. Taylor, Introduction to Functional Analysis. John Wiley, 1958

[37] M. Tsuji, Potential Theory in Modern Function Theory, Maruzen Publ. Co., Tokyo, 1959

[38] F. Ursell, On the heaving motion of a circular cylinder on the surface of a fluid, Quart. J. Mech. Appl. Math. II 2, 218-231 (1949)

[39] F. Ursell, On the rolling motion of cylinders in the surface of a fluid, Quart. J. Mech. Appl. Math. II 3, 335-353 (1949)

[40] F. Ursell, Short surface waves due to an oscillating immersed body, Proc. Roy. Soc. London Ser. A 220, 90-103 (1953)

[41] F. Ursell, The decay of the free motion of a floating body, J. Fluid Mech. 19, 305-319 (1964)

[42] F. Ursell, A problem in the theory of water waves, Numerical Solution of Integral Equations, edited by L. M. Delves and J. Walsh, Clarendon Press, 1974

[43] M. Vullierme-Ledard, Vibrations engendrées par les oscillations forcées d'un corps rigide immergé dans un fluide incompressible présentant une surface libre, C. R. Acad. Sci. Paris A 296, 611-614 (1983)

[44] S. E. Warschawski, On differentiability at the boundary in conformal mapping, Proc. Amer. Math. Soc. 12, 614-620 (1961)

[45] S. E. Warschawski and G. E. Schober, On conformal mapping of certain classes of Jordan domains, Arch. Rational Mech. Anal. 22, 201-209 (1966)

[46] J. V. Wehausen and E. V. Laitone, Surface waves, Encyclopedia of Physics, vol. IX, Springer-Verlag, 1960 , pp. $446-778$

[47] J. V. Wehausen, Methods for boundary-value problems in free surface flows, David Taylor Naval Ship Research and Development Center, Report 4622 (1974) 